\title{
Do People Agree on How Positive Emotions Are Expressed? A Survey of Four Emotions and Five Modalities Across 11 Cultures
}

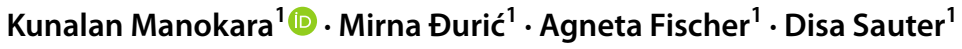

Accepted: 3 June 2021 / Published online: 22 July 2021

(c) The Author(s) 2021

\begin{abstract}
While much is known about how negative emotions are expressed in different modalities, our understanding of the nonverbal expressions of positive emotions remains limited. In the present research, we draw upon disparate lines of theoretical and empirical work on positive emotions, and systematically examine which channels are thought to be used for expressing four positive emotions: feeling moved, gratitude, interest, and triumph. Employing the intersubjective approach, an established method in cross-cultural psychology, we first explored how the four positive emotions were reported to be expressed in two North American community samples (Studies $1 \mathrm{a}$ and $1 \mathrm{~b}: n=1466$ ). We next confirmed the crosscultural generalizability of our findings by surveying respondents from ten countries that diverged on cultural values (Study 2: $n=1826$ ). Feeling moved was thought to be signaled with facial expressions, gratitude with the use of words, interest with words, face and voice, and triumph with body posture, vocal cues, facial expressions, and words. These findings provide cross-culturally consistent findings of differential expressions across positive emotions. Notably, positive emotions were thought to be expressed via modalities that go beyond the face.
\end{abstract}

Keywords Positive emotions $\cdot$ Expressions $\cdot$ Multiple modalities $\cdot$ Intersubjective approach · Culture

\section{Introduction}

Most people have a keen awareness of how others express their feelings, particularly for negative emotions (Rozin et al., 2005). When a friend seems sad (Yoo \& Noyes, 2016), or when a colleague gets angry (Tiedens, 2001), we are often able to infer how they feel. Yet our current understanding of how positive emotions are expressed is much less developed, because work tends to either focus on one or two positive emotions (e.g., Tracy \& Robins, 2007; Vritcka et al., 2014), or to examine emotions within a particular modality or two

Kunalan Manokara

k.manokara@uva.nl

1 Department of Social Psychology, University of Amsterdam, Amsterdam, The Netherlands 
(e.g., Dael et al., 2012a, 2012b; Ekman et al., 1987; Laukka, 2005). However, theoretical accounts suggest that positive emotions would be expected to be expressed in ways that are congruent with their functions (Kitayama et al., 2006; Sauter, 2017). The goal of this paper is to provide an empirical test of these theoretically informed predictions.

In the present research, we explore how people think that four specific positive emotions are expressed: feeling moved, gratitude, interest, and triumph. For each emotion, we study the reported use of five modalities of expression: face, voice, body, touch, and words. To examine the replicability and generalizability of our findings across cultures, we adopt a method from cross-cultural psychology: the intersubjective approach (Chiu et al., 2010). In doing so, we map out how four specific positive emotions are thought to be expressed in 11 countries characterized by divergent cultural values (Minkov \& Hofstede, 2012; Schwartz et al., 2001).

\section{Two Frameworks of Positive Emotions}

The interest in understanding positive emotions is growing with advances in the fields of affective science (Keltner, 2019), and social psychology (Fredrickson, 2001). Previous theorizing and research in which negative and positive emotions were compared has mainly focused on joy (or happiness), which is conceived as a singular emotion argued to be primarily expressed using the face (e.g., Ekman, 1992). However, recent theorizing posits that there are multiple discrete positive emotions (see Shiota et al., 2017) that are expressed through channels that go beyond the face, including postural cues (Dael et al., 2012a, 2012b), the use of words (Campos et al., 2013), displays via touch (Schirmer \& Adolphs, 2017), and vocalizations (Sauter \& Scott, 2007).

Several accounts have sought to map out how different positive emotions relate to one another and what functions they serve. Whereas none of these accounts yield straightforward predictions of how different positive emotions are expressed, we drew on this work to inform our selection of emotions as well as in the formation of exploratory initial hypotheses. Given the long-running debate in the field with regard to the universality of emotion (e.g., Russell, 1994, and reply by Ekman, 1994), we selected two frameworks that each stem from a different research tradition: the emotion families approach (Sauter, 2017) and the arousal-engagement matrix (Kitayama et al., 2006; Tsai et al., 2006). Emotion families are based on evolutionary functions that are shared across human beings (Tooby \& Cosmides, 2008), with a focus on specialised functions for different emotions. The arousalengagement framework emphasises cross-cultural differences (Boiger \& Mesquita, 2012), and posits that emotions can be classified according to underlying dimensions. Although these taxonomies are inspired by divergent lines of work, they are not mutually exclusive, because they each focus on different elements and functions. We first outline these frameworks and the predictions we derived from them, and next specify the modality-specific hypotheses for each of the four positive emotions that are the focus of the present paper.

The emotion families approach (Sauter, 2017) postulates that positive emotions can tentatively be classified into one of four clusters, which diverge in terms of evolutionary functions (for similar suggestions, see Shaver et al., 1987, and App et al., 2011). This classification scheme is informed by work relating to expressions (e.g., Simon-Thomas et al., 2009) and appraisals (e.g., Roseman, 1996). The modalities in which different positive emotions are expressed is thought to map onto the purported function of the cluster in which a given emotion is thought to belong. Epistemological emotions (e.g., interest) involve a change or shift in one's knowledge state. Congruent with the cognitive nature of such emotions, expressing 
Table 1 List of positive emotions with corresponding categories based on emotion family, physiological arousal, and social engagement, as well as predicted expressive modalities

\begin{tabular}{lllll}
\hline & Emotion family & $\begin{array}{l}\text { Physiological } \\
\text { arousal }\end{array}$ & Social engagement & Predicted modalities \\
\hline Feeling moved & Savouring & High & Other focused & Face \\
Gratitude & Prosocial & Low & Other focused & Words, voice \\
Interest & Epistemological & Low & Malleable & Words, face, voice \\
Triumph & Agency-approach & High & Self focused & Body, face, voice \\
\hline
\end{tabular}

an epistemological emotion would be likely to involve the use of higher order cognitive processes, such as language (LeDoux \& Brown, 2017). Prosocial emotions (e.g., gratitude) focus people towards the welfare of others and help foster social relationships. Expressing prosocial emotions should hence involve affiliative gestures that bring people closer together, which can be expressed through physical contact or verbal signals. Savoring emotions (e.g., feeling moved) stem from experiencing enjoyable, calming, or heartwarming stimuli. Given that such emotions are purportedly linked to unconditioned stimuli that make one feel good, expressions would be likely to include smiling. Finally, agency-approach emotions (e.g., triumph) are characterized by approach tendencies towards potential rewards. Given the need to explicitly display movement towards an objective, such emotions may be likely to involve bodily movement.

In the arousal-engagement matrix, we combine the complementary dimensions of physiological arousal (Tsai et al., 2006) and social engagement (Kitayama, et al., 2006), such that positive emotions could theoretically fit into one of four potential quadrants. Arousal refers to the extent to which an emotion elicits a heightened physiological response in an individual (Larsen \& Diener, 1992), while engagement denotes the degree to which an emotion is socially oriented and thereby brings people closer together (Kitayama et al., 2000). Based on this framework, high arousal emotions may involve the recruitment of multiple nonverbal modalities that act as amplifiers to convey the heightened arousal an expresser feels. In contrast, low arousal emotions might involve less nonverbal signals, and a greater reliance on words to express how one feels instead. Socially engaging emotions should be likely to involve the use of signals that clearly display affiliative motives - such as smiling, touch, and wordsto bring people closer together and to minimize interpersonal misunderstandings. Conversely, socially disengaging emotions, which increase social distance between oneself and others, should make less use of the abovementioned channels.

Based on these theoretical taxonomies, we selected four positive emotions that varied in terms of emotion family, arousal, and social engagement: feeling moved, gratitude, interest, and triumph. Table 1 lists the four positive emotions we selected in relation to the theoretical frameworks. We also added the modalities through which we expected each emotion to be expressed. These modalities are based on both the characteristics and functions of each of these emotions, according to the theoretical frameworks outlined above, as well as a review of the empirical literature on nonverbal expressions. In the ensuing section, we lay out the rationale for our predictions. 


\section{Nonverbal Expressions of Positive Emotions}

A growing body of empirical work has shown that some specific positive emotions have uniquely distinguishable expressive signals (Keltner \& Cordaro, 2017), occurring across multiple channels (Cowen et al., 2019; Elfenbein et al., 2007). In production studies, researchers typically examine how people express positive emotions using one or two modalities (most commonly the face, and to a lesser extent voice). Expressions can be produced spontaneously in ecologically valid scenarios (Tracy \& Matsumoto, 2008), with poses in controlled laboratory settings (Cordaro et al., 2018), or elicited with stimuli that are meant to induce specific emotions (Levenson et al., 1991). The expressive cues, such as facial muscle movements or vocalizations, are then empirically mapped using established coding schemes (Dael et al., 2012a, 2012b; Ekman et al., 2002) and automatic detection software using algorithms (Krumhuber et al., 2019; Schuller \& Schuller, 2020). A broad range of positive emotions have been found to have specific nonverbal cues (for a review, see Sauter, 2017). For example, amusement is expressed with an open-jaw smile (Ambadar et al., 2009) and vocalizations with multiple amplitude onsets (Sauter et al., 2010), while awe is signaled with raised eyebrows, eye-widening, parted lips (Campos et al., 2013), and visible inhalations (Shiota et al., 2003).

Yet, little research has systematically compared across modalities (see Kessous et al., 2010, and Schirmer \& Adolphs, 2017). Our present understanding of positive emotional expressions is based on scattered bodies of work, and many modalities have yet to be considered in relation to specific positive emotions. The empirical findings we review, and our hypotheses for the present research, are consequently constrained by the limitations of this literature. With regards to our four selected positive emotions (feeling moved, gratitude, interest, triumph), some empirical evidence has been put forth that allows predictions to be made about the non-verbal cues used to express each of them. We introduce each of these emotions in turn, and outline the predictions we made for each, along with theoretical and empirical rationales for our hypotheses. In addition, where applicable, we also distinguish between signals and signs (see Sauter \& Russell, 2020). Signals are expressive behaviors that are inherently communicative; they purposefully (though not necessarily consciously) convey social information to observers (Fridlund, 1994). In contrast, signs are expressions that are simply "given off" (Goffman, 1959); neither communicative functions nor intentions are necessary components of signs.

Feeling moved is an intense emotion that is triggered when partaking in (or observing) communal sharing relationships, such as an unexpected reunion (Cova \& Deonna, 2014). This emotion is thought to be mixed in terms of valence (e.g., bittersweet), in that mostly positive but also negative feelings are elicited when feeling moved (Menninghaus et al., 2015; Vuoskoski \& Eerola, 2017). Possibly as a reflection of this negative valence, some empirical evidence points to increased muscular activity around the eyes (e.g., corrugator) when people feel moved (Wassiliwizky et al., 2017a, 2017b; but see Zickfeld et al., 2020). Robust cross-cultural evidence also suggests that feeling moved is displayed and identified via tear droplets and crying (Schubert et al., 2018; Seibt et al., 2018; Zickfeld et al., 2019b). While other modalities have also been suggested to be involved in the expression of feeling moved, such as vocalisations and hand gestures (Fiske et al., 2019; Zickfeld et al., 2019a), these modalities have not consistently been found across studies. The most robust empirical evidence has been found for tears.

In terms of the arousal-engagement matrix, feeling moved is a high-arousal highengagement positive emotion. Clear communicative signals are to be expected for 
conveying feeling moved in order to bring others closer to the expresser. This is consistent with empirical work indicating that tears signal a desire for help, and trigger approach orientations in others (Gracanin et al., 2018). In the emotion family framework, being moved is considered a savoring positive emotion. Savoring emotions typically have quite clear displays on the face (Sauter, 2017). In sum, both theoretical and empirical evidence led us to postulate that the face should be the main modality through which feeling moved is expressed.

Gratitude is defined as a feeling of thankfulness and indebtedness for the positive actions and contributions of another person or group (Algoe, 2012). Much of the work on gratitude has focused on verbal rather than nonverbal expressions (Algoe \& Haidt, 2009; Algoe et al., 2013), with research showing that when indebted, people express being thankful with words of appreciation (Williams \& Bartlett, 2015). There are also findings pointing to gratitude being expressed via handshakes, meaning that touch could be employed in some contexts (Hertenstein et al., 2009). A clear facial expression for gratitude has however yet to be surfaced (Campos et al., 2013), and given the scant attention paid to vocalisations of gratitude (see also Yoshimura \& Berzins, 2017), it remains theoretically possible that people may reduce the volume of their voice when they feel grateful.

Gratitude is a low-arousal high-engagement positive emotion. It is thus similar to feeling moved in terms of triggering social orientations. However, gratitude displays would be expected to be less intense in nature, reflecting the comparatively lower state of arousal of the expresser. In term of emotion families, gratitude is a prosocial emotion, which entails communication via touch in order to stimulate closeness and social attachment. This aligns with empirical work as described above (App et al., 2011). However, expressing gratitude through touch (e.g., clasping someone's hand) also intrudes on the physical space of the perceiver, and so would only be appropriate to use between people who already have a close relationship with one another (Lee \& Guerrero, 2001). Taking theory and the contextual nature of empirical findings into account, we therefore expected only words and the voice to be used for expressing gratitude.

Interest is conceptualised as a feeling that arises when new and relevant stimuli are encountered in the environment (Silvia, 2008). It is a multi-faceted emotion, such that expression modalities may depend on the domain of interest (Silvia, 2005). Past work has demonstrated interest to be expressed using a quickened speech rate, and a widened vocal frequency range (Banse \& Scherer, 1996). The use of phrases and questions also signal a keenness to engage (Silvia, 2008), indicating that both the voice and words could be key communicative channels for expressing interest. For the facial expression of interest, both signals (constriction of the eyebrows to communicate a deep concentration on a specific topic: Campos et al., 2013; smiling: Mortillaro et al., 2011) and signs (parted lips: Reeve, 1993; widened eyes: Shiota et al., 2003) are postulated to be to be involved. A case has also been made for a body movement sign associated with interest, namely, leaning forward (Dael et al., 2012a, 2012b; Dukes et al., 2017).

Interest is thought to be a low-arousal emotion. This would suggest that interest is not likely to be expressed using the full range of body movements, which would be more likely to characterise emotions that are more intense. In terms of engagement, interest is thought to be malleable; being interested draws one's attention inwards towards a specific topic (Sung \& Yih, 2016), but at the same time, one could also share their interest with others (Yoon et al., 2012; Rime, 2009). The epistemological nature of interest points to the potential use of higher order cognitive processes like language, and so words could be an important communicative tool for expressing interest. Given that a direct comparison between modalities has yet to be made for interest, we postulated the use of modalities that both 
empirical work and theorising concur with. As such, words, the face, and voice, were all expected to be used to express interest.

Triumph is the feeling elicited upon victory (Matsumoto \& Hwang, 2012). Empirical work has suggested that in the immediate aftermath of winning, specific signals emerge: people adopt a straightened body posture with the chest protruding, and make guttural sounds signalling their victory (Tracy \& Matsumoto, 2008). There is also evidence from two divergent cultural samples examining athletes immediately post victory, which found that winners tended to show open mouthed smiles and upward head tilting (Hwang \& Matsumoto, 2014). Even in work suggesting that victory displays are regulated so as to spare perceivers' feelings, an expansive body posture comes through as a partially suppressed signal related to winning (Van Osch et al., 2019).

From a theoretical standpoint, triumph is deemed a high-arousal low-engagement emotion. Given high arousal, its expressions should involve the recruitment of multiple expressive channels to demonstrate a larger social presence via expansiveness of the self (App et al., 2011). The dominance focused nature of triumph signals highlights the socially disengaging nature of this emotion (Kalokerinos et al., 2014), and suggests that multiple channels should be involved in its expression. Similar expressive patterns are expected based on triumph being an agency-approach emotion. To signal one's agency and possibly even power, triumph should involve body postures and head movements that seek to make the self look taller and larger (Hwang et al., 2016). Both theory and empirical work thus point to the hypothesis that triumph should be expressed via body movements, facial expressions, and the voice.

\section{Emotion Expressions Across Cultures}

A major caveat to the patterns of expression described above is that the degree to which they generalize to people from most parts of the world is at present unestablished. Much of the empirical research on emotional expressions has thus far been conducted with North American samples (see Keltner \& Cordaro, 2017), and even genuine attempts at cross-cultural work are often hampered by the fact that most respondents are university students. Student samples share many common characteristics regardless of which part of the world they come from (Henrich et al., 2010) and are typically not representative of the populations they are thought to reflect. While some researchers have recruited community samples to draw a contrast between modernized and culturally isolated populations, such lines of work have tended to either focus on one specific positive emotion (e.g., Tracy \& Robins, 2008), or a particular modality in isolation (e.g., Ekman et al., 1987; Sauter et al., 2010). The need to study community samples across a broad range of cultures, while examining various modalities of expression for different positive emotions, remains a worthwhile endeavour for the advancement of theory in affective science.

For the present research, we adopted an established method from cross-cultural psychology to examine self-reported expressions of positive emotions: the intersubjective approach (Chiu et al., 2010). When applied to emotion expressions, the intersubjective approach entails asking people how other members of their culture in general express specific positive emotions (feeling moved, gratitude, interest, triumph), with commonly used modalities presented as response options (face, voice, body, touch, words).

Self-report instruments provide many advantages for cross-cultural work, especially when contrasted with classic methods from production studies. Many of the existing tools used in expression research are not only costly and time intensive, but also involve the 
practical constraints associated with setting up on-site data collection in divergent cultural contexts. As a means of navigating such difficulties, other researchers too have employed variants of self-report: respondents choose from photographs that depict various stages of an expression (Matsumoto, 1990; Van Osch et al., 2019), indicate on a mannequin or avatar which parts of the body are most implicated when an emotion is felt (Nummenmaa et al., 2014; Van Cappellen \& Edwards, 2020), or recollect emotional experiences and textually describe all components they associate with each specific emotion, including expressions (Campos et al., 2013). These self-report formats facilitate cross-cultural research, because surveys can be conducted wholly online, and thus be used to reach community samples with relative ease and at lower cost than traditional lab studies.

The intersubjective approach deals with two common methodological concerns associated with self-reported emotion expressions: the expectation that participants have accurate insight into how they express different emotions, and the assumption that aggregated individual responses can be taken as a valid reflection of a cultural group's response patterns. By using the intersubjective approach, we measure expressions from the perspective of the perceiver, thereby capturing estimates of expressions from commonly encountered targets in everyday life. Such items mitigate the need for introspective evaluation by shifting the focus away from one's own behaviors to observed displays in others. Furthermore, by presenting categorical response options with clear and familiar labels, this approach eliminates culturally-dependent response biases that may occur on Likert-type scales (Van Herk et al., 2004). Because this approach is based on the principle of shared consensus (Wan et al., 2010), culture-level conclusions are drawn based on agreement between raters, rather than simply assuming a given sample's demographic features and response patterns to be representative of a larger nation unit.

In sum, existing theorizing and empirical work suggest that positive emotions are nonverbally expressed through several different channels, yet to date studies have tended to examine only singular (or maximally two) modalities at a time. Here, we sought to provide a first step towards systematically mapping out which modalities are associated with particular positive emotions across countries that differ on cultural values (Minkov \& Hofstede, 2012; Schwartz et al., 2001).

\section{Current Research}

In the present research, we use intersubjective judgments to map out how several positive emotions are thought to be expressed across a wide range of cultures. Specifically, we examine how people perceive others in their culture to express four distinct positive emotions, selected based on the emotion families approach (Sauter, 2017) and the arousalengagement matrix (Kitayama et al., 2006; Tsai et al., 2006). In three studies, we examined peoples' perceptions of how others in their culture express feeling moved, gratitude, interest, and triumph. Study 1a was exploratory and mapped out which modalities were most frequently thought to be associated with expressing feeling moved, gratitude, interest, and triumph in the U.S.A. We selected emotions that have been corroborated by past research to have some degree of identifiable expressions in North America (Cowen \& Keltner, 2020). This also allowed for a critical evaluation of the intersubjective rating method, where our obtained findings should be in alignment with previous research. Study $1 \mathrm{~b}$ was a pre-registered replication study with a separate community sample from the U.S.A., which sought to test whether the removal of emotion definitions would shift participants' responses (see https://osf.io/yrpa4/?view_only=3d8c542ebdfd47ffba126ca96e5ac142). 
Study 2 was cross-cultural and confirmatory, and also pre-registered (see https://osf.io/ p7je9/?view_only=8a8c076d0c974e6bb6038df06df2b892). We examined the degree to which our initial predictions would hold across a set of national samples that varied on cultural values. We broadly sampled from multiple regions of the world, where respondent also spoke varying languages (see Table 5 for further information). We here recap our initial exploratory hypotheses for each positive emotion. By predicting that specific modalities would be most frequently reported, we expected those particular modalities to be selected significantly more often than all of the other expressive channels.

\section{Hypotheses}

H1 (feeling moved) We expected people to report that feeling moved (Menninghaus et al., 2015 ) would be most frequently expressed on the face as compared to the other modalities.

H2 (gratitude) We predicted that people would report that gratitude (Algoe et al., 2013) is most regularly expressed using words and the voice, when contrasted against the other modalities.

H3 (interest) We hypothesised that people would report that interest (Silvia, 2008) is most frequently expressed using one's face, words, and voice, as compared to the other modalities.

H4 (triumph) We predicted that people would think triumph (Tracy \& Matsumoto, 2008) is most frequently expressed using the face, body movement, and voice, when contrasted against the other modalities.

\section{Ethics Statement}

All studies reported in this paper received ethical approval from the Department of Psychology at a large European university. For fully anonymized data files, materials, and analysis script, see: https://osf.io/szah4/?view_only=3270f24f3c704f34b42d88f574930fa6

\section{Study 1a: Exploring Patterns in the U.S.A.}

Study 1a sought to map out which modalities were most frequently reported to occur as expressions of four specific positive emotions in a community sample from the U.S.A.

\section{Method}

\section{Participants}

As part of two larger studies 1015 U.S.A. citizens (64.14\% male; $73.69 \%$ White; all residing in the U.S.A. at the time of the study) were recruited via Amazon Mechanical Turk (age range 18-77 years; $M_{\text {age }}=36.46, S D_{\text {age }}=10.79$ ). Participants were paid approximately US $\$ 1$ for the 10-min survey. Given the exploratory nature of the study, we aimed to assemble a dataset with a subject-to-item ratio of at least 10:1 (Everitt, 1975). We achieved a 
Table 2 Definitions of positive emotions provided to the participants

\begin{tabular}{ll}
\hline Emotion & Definition \\
\hline Feeling moved & $\begin{array}{r}\text { The feeling when you encounter something very beautiful, meaningful, or bittersweet. } \\
\text { Tears well up in your eyes and you feel overcome with warm feelings }\end{array}$ \\
Gratitude & $\begin{array}{r}\text { The feeling when you think that someone has gone out of their way to do something good } \\
\text { or nice for you. You have the urge to do something back and get closer to this person }\end{array}$ \\
Interest & $\begin{array}{r}\text { The feeling when you encounter something new and relevant that you do not immediately } \\
\text { understand. You have the urge to find out more about it }\end{array}$ \\
Triumph & The feeling of release and a great joy, after a successful ending of a struggle or contest
\end{tabular}

ratio of 42:1 with our final dataset, thus a robust sample size. Furthermore, sensitivity analyses using $\mathrm{G}^{*}$ Power Version 3.1.9 (Faul et al., 2009) indicated our sample to be adequately powered at $80 \%$ for detecting small effects in frequency distribution models such as Chisquare analyses $(W=0.11)$.

\section{Materials}

Participants were first shown a list of the four positive emotions and provided with definitions for each emotion (shown in Table 2), derived from commonly used definitions in the literature (e.g., Algoe et al., 2013; Cova \& Deonna, 2014; Silvia, 2008; Tracy \& Matsumoto, 2008). The participants were then asked to think about how members of their nation in general express each positive emotion, and were asked to select options denoting various modalities of expression: (1) with the voice, (2) on the face, (3) using body movement, (4) with words, (5) via touch, (6) in other ways. Participants could select all options that applied. ${ }^{1}$

\section{Procedure}

Participants made judgements about each of the four positive emotions separately, with the order of emotions randomized. After all emotion items were completed, demographic information was collected and participants were debriefed and paid.

\section{Results}

\section{Modality Use Per Emotion}

To calculate percentage scores for the selection of each modality, we divided the number of participants who selected the modality by the overall number of participants. These scores thus reflect the percentage of participants that think a given emotion is expressed via that modality. Table 3 lists these percentage scores per emotion.

\footnotetext{
1 Participants were not allowed to skip any question, meaning they could not select no modalities for a given emotion. If participants selected Option 6, a free response box was also provided. However, few participants used it. While we included the category Other in our analyses to reflect the options offered to the participants, we do not report or interpret the specific differences between this and other modalities in the text, as there were generally few responses in this category.
} 
Table 3 Reported expressions (\% with $95 \%$ confidence intervals) for each emotion per modality in the U.S.A. (Study 1a)

\begin{tabular}{cllllll}
\hline & Voice & Face & Body & Words & Touch & Other \\
\hline Feeling & 55.67 & $\mathbf{7 4 . 4 8}$ & 45.71 & 56.65 & 28.08 & 2.07 \\
moved & {$[52.61,58.73]$} & {$[71.80,77.16]$} & {$[42.65,48.77]$} & {$[53.60,59.70]$} & {$[25.32,30.84]$} & {$[1.19,2.95]$} \\
Gratitude & $\mathbf{6 4 . 8 3}$ & 55.27 & 44.33 & $\mathbf{7 4 . 9 8}$ & 37.04 & 4.43 \\
& {$[61.89,67.77]$} & {$[52.21,58.33]$} & {$[41.27,47.39]$} & {$[72.32,77.64]$} & {$[34.07,40.01]$} & {$[3.16,5.70]$} \\
Interest & $\mathbf{6 2 . 1 7}$ & $\mathbf{6 5 . 9 1}$ & 43.15 & $\mathbf{6 6 . 6 0}$ & 25.12 & 1.77 \\
& {$[59.19,65.15]$} & {$[62.99,68.83]$} & {$[40.10,46.20]$} & {$[63.70,69.50]$} & {$[22.45,27.79]$} & {$[0.96,2.58]$} \\
Triumph & $\mathbf{6 7 . 0 0}$ & $\mathbf{6 3 . 5 5}$ & $\mathbf{6 9 . 3 6}$ & 62.86 & 20.20 & 1.77 \\
& {$[64.11,69.89]$} & {$[60.59,66.51]$} & {$[66.52,72.20]$} & {$[59.89,65.83]$} & {$[17.73,22.67]$} & {$[0.96,2.58]$} \\
\hline
\end{tabular}

Numbers indicate the percentage of participants who selected a given modality as a channel used to express that emotion. The percentages corresponding to the hypothesized modalities are indicated in bold. Confidence intervals for the Other modality category are potentially unreliable due to the low values

Given the overlapping nature of our response options, the most commonly used chisquare techniques for comparing frequencies were unsuitable due to dependency in the data, which violates one of their key assumptions. Therefore, we utilised a method from the chi-square family of tests appropriate for overlapping response options: Cochran's Q test, an extension of McNemar's test for three or more related proportions (Cochran, 1950; see also Mielke et al., 2007). We conducted separate Cochran's Q tests for each emotion by comparing the proportion of participants who reported different modality use within each positive emotion. ${ }^{2}$ We set a stringent significance threshold for these models of $p<0.001$ to account for the number of models computed. ${ }^{3}$ These omnibus tests were used to determine whether there was a difference between the modalities overall for each emotion; we investigated differences between specific modalities within each emotion by conducting pairwise McNemar's tests with p-values Bonferroni-adjusted for 15 comparisons (the number of possible modality pairings). Pairwise comparisons for all modalities for each emotion are reported in the Supplementary Materials (ESM Pg. 2).

Feeling moved The difference between modalities was found to be significant for expressions of feeling moved, $Q(5)=1449.43, p<0.001, R=0.219$. As predicted, Americans thought feeling moved was more frequently expressed on the face (reported by $74.48 \%$ participants) as compared to all other modalities (28.08-56.65\%).

Gratitude A significant difference was surfaced between selected modalities for expressing gratitude, $Q(5)=1408.15, p<0.001, R=0.206$. Consistent with our prediction, participants reported that they thought gratitude was more frequently expressed with words (74.98\%) and the voice (64.83\%) as compared to face, body, and touch (37.04-55.27\%).

Interest The difference between selected modalities was found to be significant for expressing interest, $Q(5)=1520.52, p<0.001, R=0.233$. As predicted, Americans

\footnotetext{
${ }^{2}$ We conducted the Cochran's Q tests, and the follow-up McNemar's tests, using R version 3.6.2 (R Core Team, 2019). We used the R packages RVAideMemoire (Hervé, 2020), and rcompanion (Mangiafico, 2020).

${ }^{3}$ In reporting effect sizes for the Cochran's Q test, we used the chance-corrected measure (R) introduced by Berry, Johnston, and Mielke (2007).
} 


\section{(A) Feeling Moved}

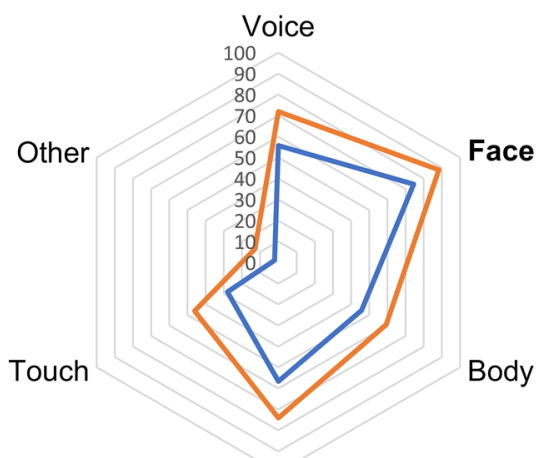

Words
(B) Gratitude

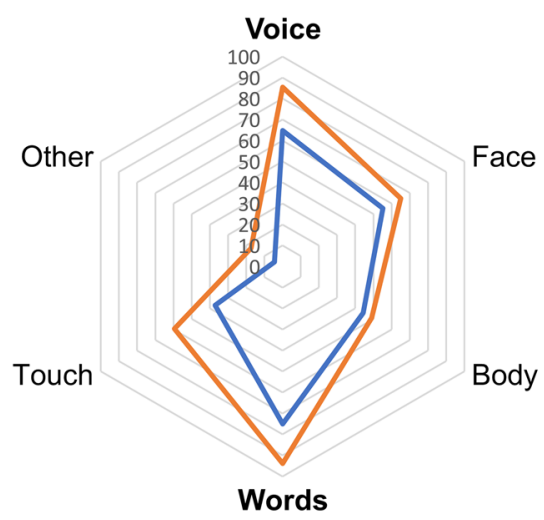

(D) Triumph

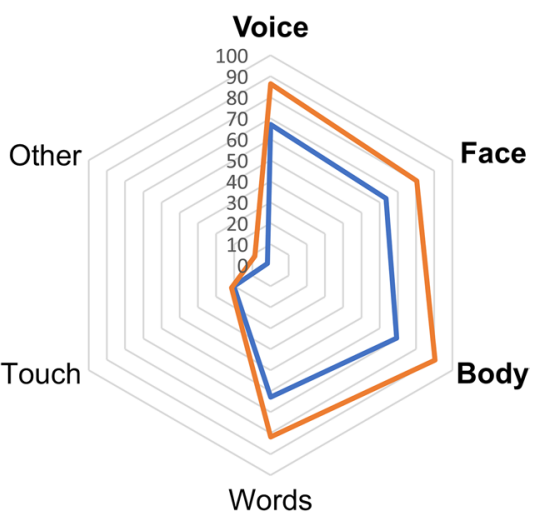

- Study 1a (with definitions)

- Study $1 b$ (without definitions)

Fig. 1 Radial plots showing the percentage of participants reporting the use of different modalities to express a Feeling Moved, b Gratitude, c Interest, and d Triumph in the United States of America (Study 1a Sample and Study 1b Sample). Note: The hypothesized modalities are indicated in bold

reported that interest was more frequently expressed with words $(66.60 \%)$, the face (65.91\%), and the voice $(62.17 \%)$, compared to touch $(25.12 \%)$ and bodily cues $(43.15 \%)$.

Triumph A significant difference was found between selected modalities for triumph expressions, $Q(5)=1848.93, p<0.001, R=0.279$. As predicted, triumph was thought to be more frequently expressed via the body $(69.36 \%)$, voice $(67.00 \%)$, and face $(63.55 \%)$ as compared to touch $(20.20 \%)$. Contrary to predictions, however, the American participants thought words (62.86\%) were equally commonly occurring as expressions of triumph as were facial and vocal expressions (see Fig. 1 in Study 1b). 


\section{Discussion}

In Study 1a, we mapped out how Americans think four specific positive emotions are expressed. Largely in line with our initial predictions, participants reported that feeling moved is conveyed primarily using the face, gratitude by what someone says and how it is said, interest is communicated using facial expressions, words, and the voice, while triumph was expressed using the face, voice, body, and-contrary to our prediction-words. We interpreted this unexpected finding to be a culture-specific pattern that reflects U.S.A. culture, where verbal expressions of triumph are often encouraged (Hwang \& Matsumoto, 2014; Van Osch et al., 2013).

While nearly all of the findings support our predictions, we acknowledge a potential shortcoming of our method. By providing definitions for emotions, we could have unintentionally guided participants to select particular modalities. To rule out this alternative explanation, we conducted a pre-registered replication study with a new sample of participants from the U.S.A.

\section{Study 1 b: Replication Study Without Emotion Definitions in the U.S.A.}

Study 1b (https://osf.io/yrpa4/?view_only=3d8c542ebdfd47ffba126ca96e5ac142) was conducted to address concerns regarding the guiding of participants with emotion definitions. We recruited another community sample from the U.S.A. and applied the same procedure as Study 1a-however, without providing emotion definitions.

\section{Method}

\section{Participants}

As part of a larger study, 451 U.S.A. citizens (46.34\% male; $79.60 \%$ White; all residing in the U.S.A. at the time of the study) were recruited via Amazon Mechanical Turk (age range 18-75; $M_{\text {age }}=34.09, S D_{\text {age }}=11.53$ ). Participants were paid approximately US $\$ 3.40$ each. The sample size was determined by the data collection for a larger study, but sensitivity analyses using indicated our sample to be adequately powered at $80 \%$ for detecting small to medium effects in frequency distribution models such as Chi-square analyses $(W=0.17)$.

\section{Materials and Procedure}

All materials and the procedure were identical to Study 1a, with the exception of emotion definitions. The participants were thus not shown definitions of the emotions at any point. 
Table 4 Reported expressions (\% with $95 \%$ confidence intervals) for each emotion per modality in the U.S.A. (Study 1b)

\begin{tabular}{ccccccc}
\hline & Voice & Face & Body & Words & Touch & Other \\
\hline Feeling & 71.84 & $\mathbf{8 8 . 2 5}$ & 59.20 & 74.06 & 46.12 & 12.86 \\
moved & {$[67.69$,} & {$[85.28$,} & {$[54.66$,} & {$[70.01$,} & {$[41.52$,} & {$[9.77,15.95]$} \\
& $75.99]$ & $91.22]$ & $63.74]$ & $78.11]$ & $50.72]$ & \\
Gratitude & $\mathbf{8 5 . 3 7}$ & 64.97 & 49.00 & $\mathbf{9 3 . 7 9}$ & 59.42 & 17.29 \\
& {$[82.11$,} & {$[60.57$,} & {$[44.39$,} & {$[91.56$,} & {$[54.89$,} & {$[13.80,20.78]$} \\
& $88.63]$ & $69.37]$ & $53.61]$ & $96.02]$ & $63.95]$ & \\
Interest & $\mathbf{7 8 . 2 7}$ & $\mathbf{8 3 . 1 5}$ & 58.54 & $\mathbf{8 8 . 4 7}$ & 39.91 & 8.87 \\
& {$[74.46$,} & {$[79.70$,} & {$[53.99$,} & {$[85.52$,} & {$[35.39$,} & {$[6.25,11.49]$} \\
& $82.08]$ & $86.60]$ & $63.09]$ & $91.42]$ & $44.43]$ & \\
Triumph & $\mathbf{8 6 . 4 7}$ & $\mathbf{8 0 . 4 9}$ & $\mathbf{9 0 . 4 7}$ & 81.82 & 21.51 & 8.65 \\
& {$[83.31$,} & {$[76.83$,} & {$[87.76$,} & {$[78.26$,} & {$[17.72$,} & {$[6.06,11.24]$} \\
& $89.63]$ & $84.15]$ & $93.18]$ & $85.38]$ & $25.30]$ & \\
\hline
\end{tabular}

Numbers indicate the percentage of participants who selected a given modality as a channel used to express that emotion. The percentages corresponding to the hypothesized modalities are indicated in bold. Confidence intervals for the Other modality category are potentially unreliable due to the low values

\section{Results}

\section{Modality Use Per Emotion}

Following the procedure from Study 1a, the number of participants who selected the modality was divided by the overall number of participants to calculate percentage scores for the selection of each modality. They are listed per emotion in Table 4, with $95 \%$ confidence intervals.

To test whether the results from Study 1a would replicate, we used the same analytic approach with separate Cochran's Q tests for each emotion. Pairwise comparisons between all modalities for all emotions can be found in the Supplementary Materials (ESM Pg. 3).

Feeling Moved We found a significant difference between modalities selected for expressing feeling moved, $Q(5)=682.78, p<0.001, R=0.242$. In line with our prediction, Americans in this sample reported feeling moved to be more frequently expressed on the face (reported by $88.25 \%$ participants) than via any other modality (46.12-74.06\%).

Gratitude There was a significant difference between selected modalities for gratitude expressions, $Q(5)=749.49, p<0.001, R=0.262$. As predicted, Americans in the definition-absent sample thought gratitude was more frequently expressed with words $(93.79 \%)$ and the voice (85.37\%) as compared to face, body, and touch (49.00-64.97\%).

Interest A significant difference was found between selected modalities for expressing interest, $Q(5)=889.64, p<0.001, R=0.324$. In line with our hypothesis, participants reported that interest was more frequently expressed with words $(88.47 \%)$, the face (83.15\%), and the voice (78.27\%), compared to touch (39.91\%) and bodily cues $(58.54 \%)$.

Triumph There was a significant difference between selected modalities for expressing triumph, $Q(5)=1221.30, p<0.001, R=0.466$. As predicted, American participants in this sample reported triumph to be more frequently expressed via the body $(90.47 \%)$, voice (86.47\%), and face $(80.49 \%)$ as compared to touch $(21.51 \%)$. Contrary to predictions but 
in line with Study 1a, participants thought words (81.82\%) did not differ in frequency as expressions of triumph from facial and vocal expressions (Fig. 1).

\section{Discussion}

We replicated our key findings from Study 1a, demonstrating that even when emotion definitions were not provided to participants, the most commonly reported modalities of expressions largely matched our predictions. ${ }^{4}$ In both community samples from the U.S.A., feeling moved was reported to be conveyed primarily using the face, gratitude by what someone says and how it is said, interest via facial expressions, words, and the voice, and triumph using the face, voice, body, and words.

These findings provide consistent evidence for how respondents from one particular country think emotions are expressed. Next, we sought to test the cross-cultural robustness of our findings. The degree to which emotional expressions differ across cultures is a hotly contested topic (see Manokara \& Sauter, 2021), and we hence aimed to evaluate whether participants from various parts of the world would also think that positive emotions are expressed with similar modalities as in the U.S.A.

\section{Study 2: Cross-Cultural Comparisons}

Study 2 (https://osf.io/p7je9/?view_only=8a8c076d0c974e6bb6038df06df2b892) was conducted with two aims in mind. Firstly, we sought to examine the cross-cultural generalizability of our initial findings on the modalities thought to express feeling moved, gratitude, interest, and triumph. Secondly, we aimed to evaluate the extent of cross-cultural consistency vs variability in modalities reported for expression of different positive emotions. To achieve these aims, we sampled from ten countries that differed on geographical region, language, values, as well as other cultural dimensions (Minkov \& Hofstede, 2012; Schwartz et al., 2001). To ensure the most accurate translation across multiple languages, we chose to include emotion definitions, as is standard practice in cross-cultural work (see also Sperber et al., 1994). We tested the four hypotheses proposed in Study 1 in each of these countries.

\section{Method}

\section{Participants}

Extrapolating from the findings in Study 1, we expected medium-sized effects. ${ }^{5}$ A-priori power analyses indicated that we required 143 participants from each country to detect medium-sized effects in frequency distribution models $(W=0.30)$ at $80 \%$ power. We

\footnotetext{
${ }^{4}$ We also examined the degree to which the two samples (Study 1a and Study 1b) differed in absolute levels of endorsements of each modality, for each emotion. These results can be found in the Supplementary Materials (ESM Pg. $4-6$ ).

${ }^{5}$ An uncorrected measure of effect size for Cochran's Q ( $\eta_{\mathrm{Q}}^{2}$ : Serlin, Carr, \& Marascuilo, 1982) was used in power analyses. These statistics for the U.S.A. sample can be found in the Supplementary Materials (ESM Pg. 2).
} 


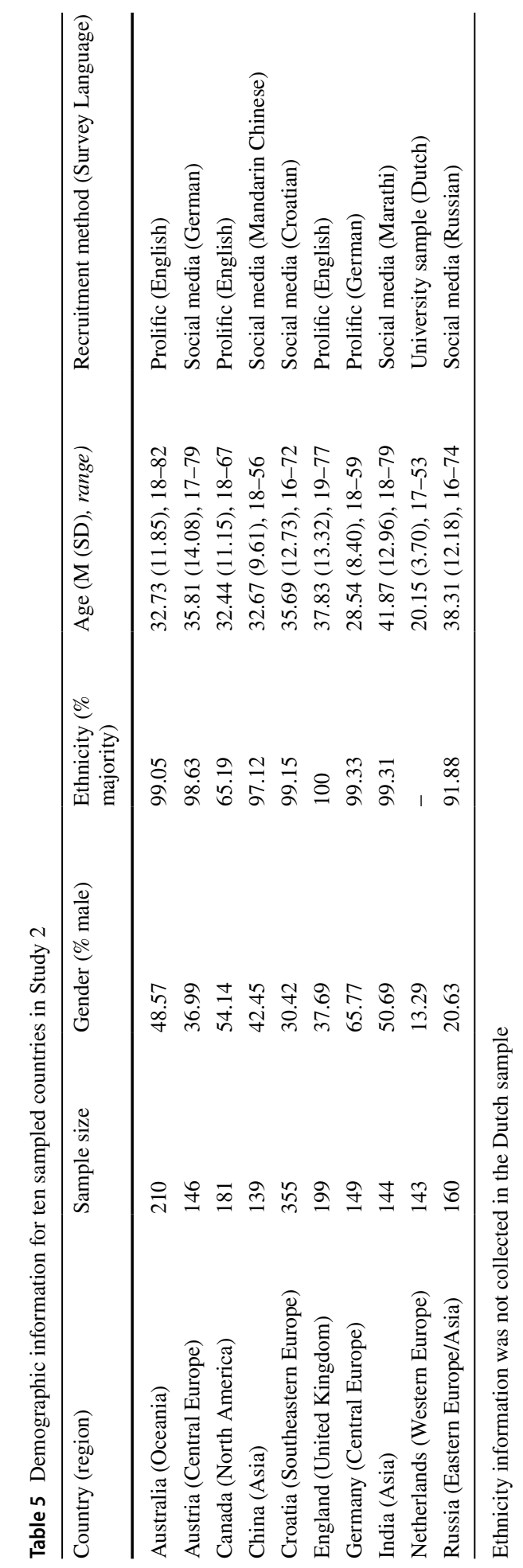


therefore sought to collect at least 150 responses per country. As part of three larger studies 1826 participants from 10 countries (39.38\% male; age range $16-82$ years; $M_{\text {age }}=33.96$, $\left.S D_{\text {age }}=12.71\right)$ were recruited through a combination of social media snowballing and paid services such as Prolific (see Table 5 for descriptive information). In line with the exclusionary criteria stated in the pre-registration, the final sample included only participants who were citizens and residents of their respective countries at the time of the study, and who provided basic demographic information (gender, age, and ethnicity).

\section{Materials and Procedure}

Using the team translation approach (Clark et al., 2017), all materials were adapted from English into the native languages of each country by two native speakers of each language. All measures and the procedure were identical to Study 1a.

\section{Results}

\section{Modality Use Per Emotion}

As in Study 1, we calculated percentage scores for the selection of each modality by dividing the number of participants who selected the modality by the overall number of participants in each country. The country-specific percentage scores and accompanying $95 \%$ confidence intervals for each emotion can be found in Table 6. Figure 2 shows an overview of reported modality use across countries for each emotion.

Analogous to the analysis approach in Study 1, we conducted separate Cochran's Q tests for each emotion, in each country. Table 7 shows the support obtained for each hypothesis across countries.

Feeling moved Significant differences between selected modalities for expressions of feeling moved were found in all countries (country-specific statistics for all emotions are reported in Table 8; pairwise comparisons for all emotions in each country can be found in the Supplementary Materials, ESM Pg. 7-16). As predicted, the face was generally the most frequently reported modality used for expressing feeling moved. This is also demonstrated in Table 6: The face was reported by a noticeably higher percentage of participants overall $(86.47 \%)$ than any other modality $(37.68-53.67 \%)$. A slight deviation from this pattern was found in China, where the face was not significantly more frequent (55.40\%) than words $(51.80 \%)$ as an expression of feeling moved.

Gratitude We found significant differences between selected modalities for gratitude displays in all countries (see Table 8). Participants in all but one culture thought words were used more frequently (reported by $88.83 \%$ participants overall) than any other modality to express gratitude, providing partial support for our hypothesis. However, against our predictions, the use of the voice $(55.81 \%$ ) did not differ in reported frequency from the use of face $(56.35 \%)$ and/or touch $(58.93 \%)$ in most cultures.

The results for gratitude also indicated several unexpected culture-specific patterns. For example, China was the only country where words were not thought to be particularly frequent. Moreover, the voice was reported rather infrequently in China, and it was the least frequently reported modality for expressing gratitude in India. In the Netherlands, touch was second only to words as the most prominent modality used to express gratitude.

Interest The differences between selected modalities for expressing interest were significant in all countries (see Table 8). In most cultures, interest was, as predicted, 


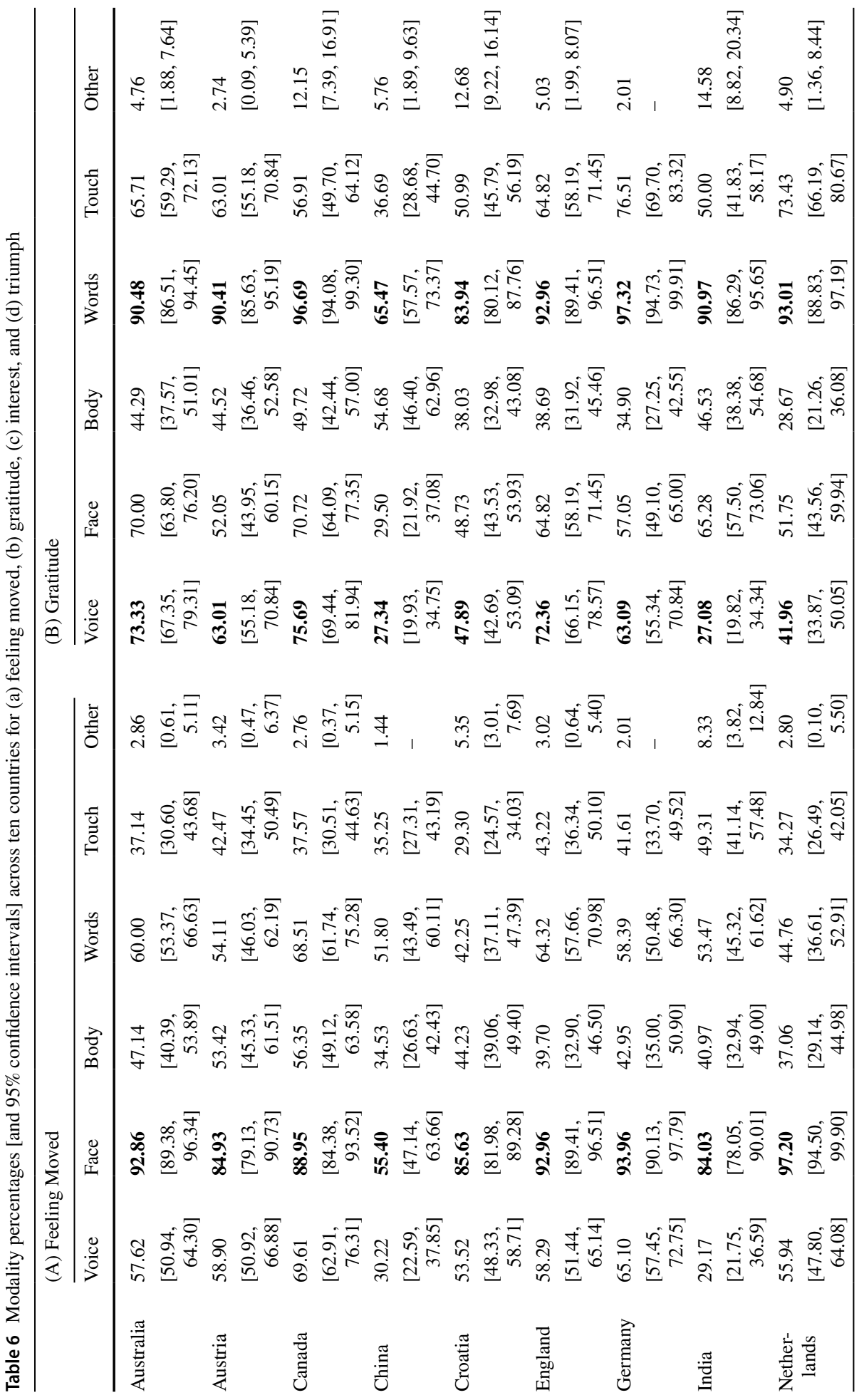




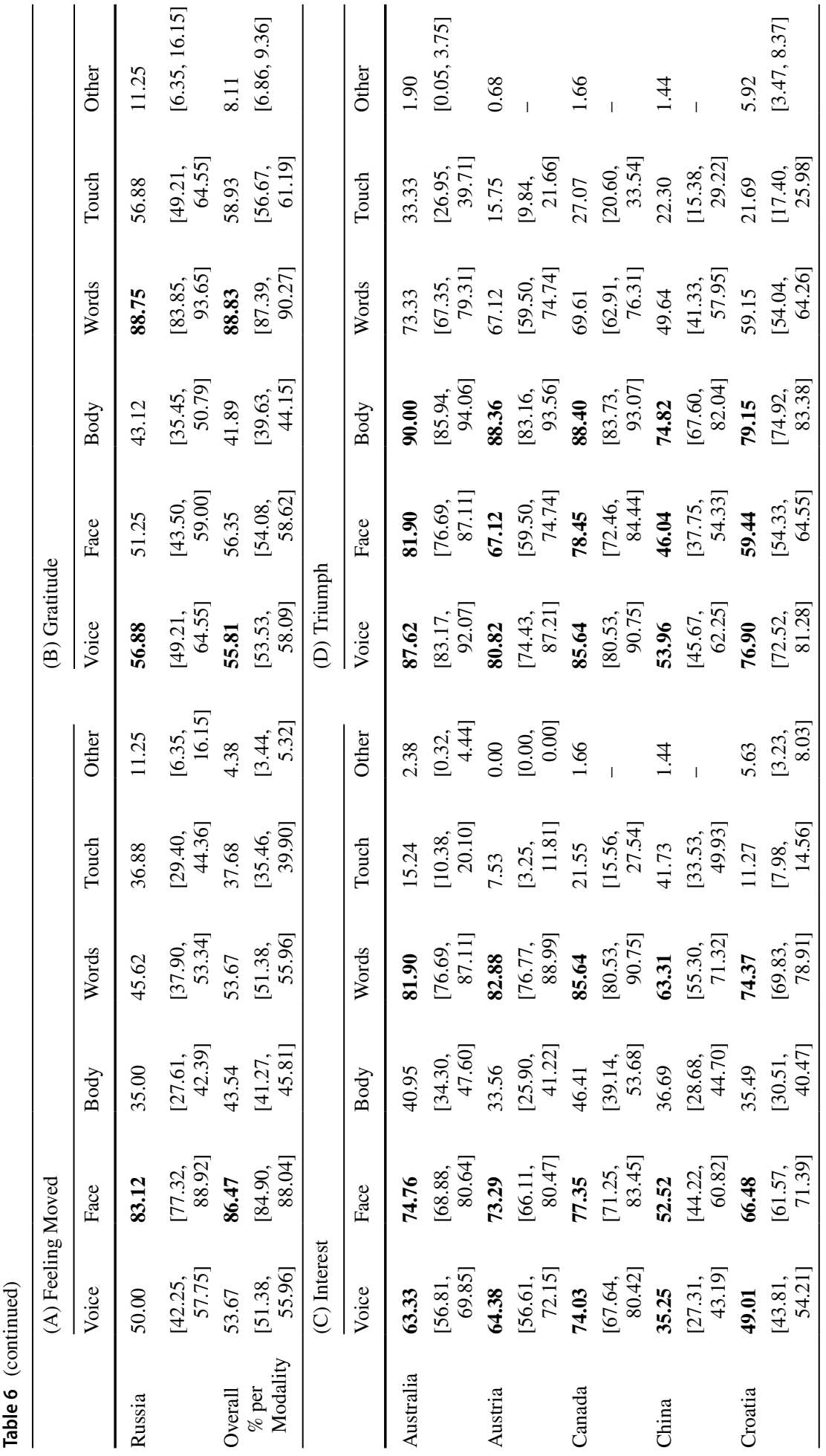




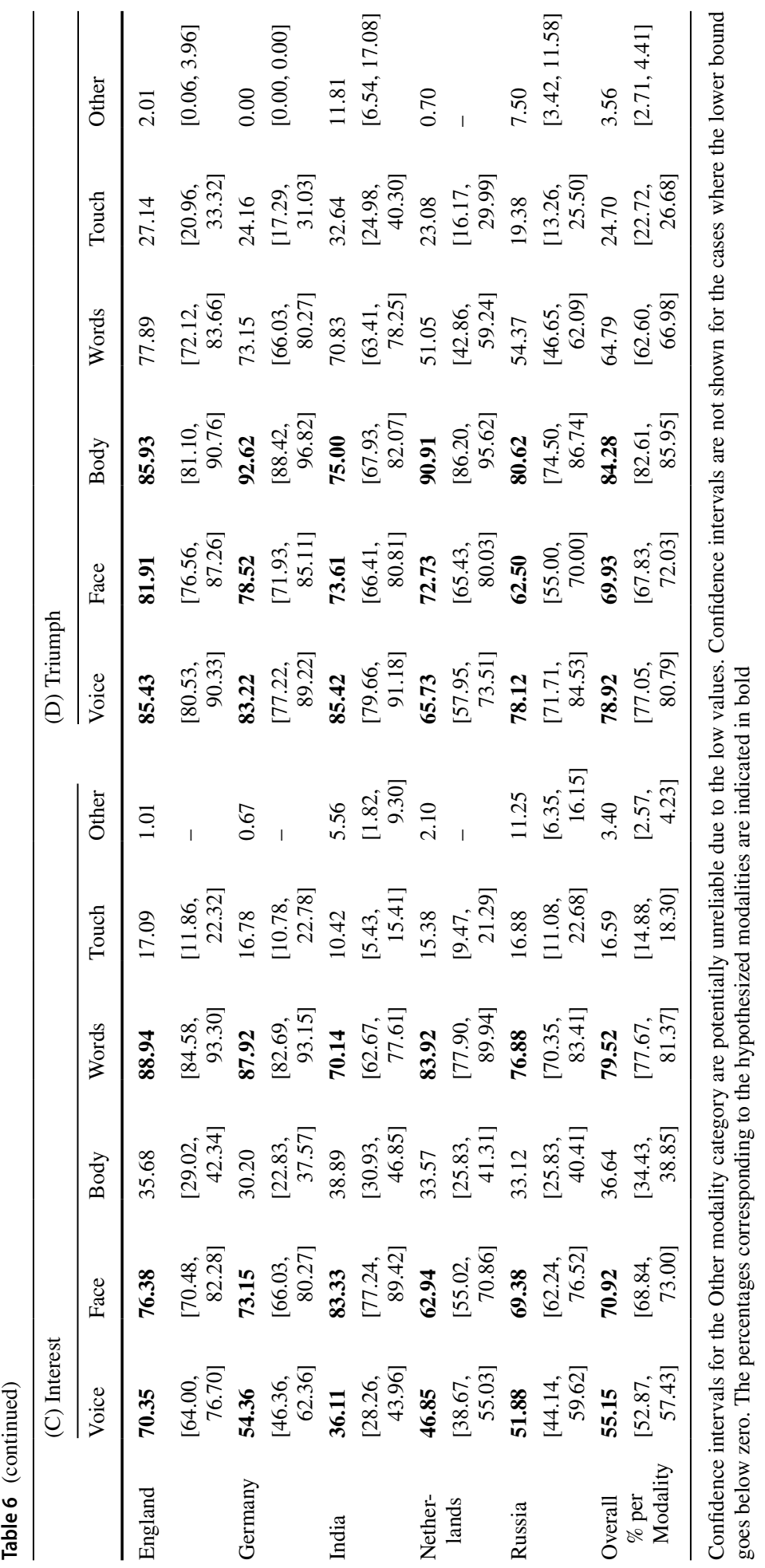




\section{(A) Feeling Moved}

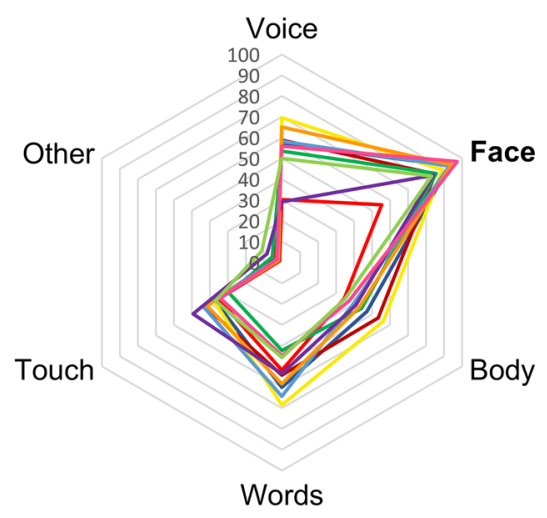

(C) Interest

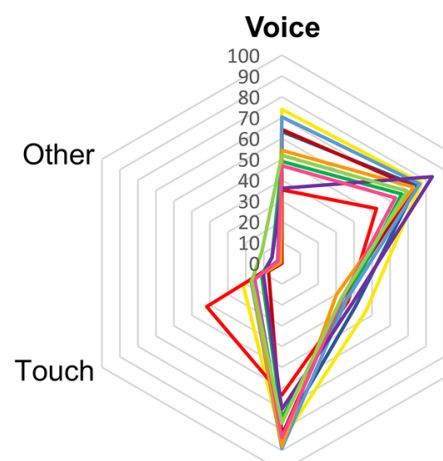

Words

\section{(B) Gratitude}

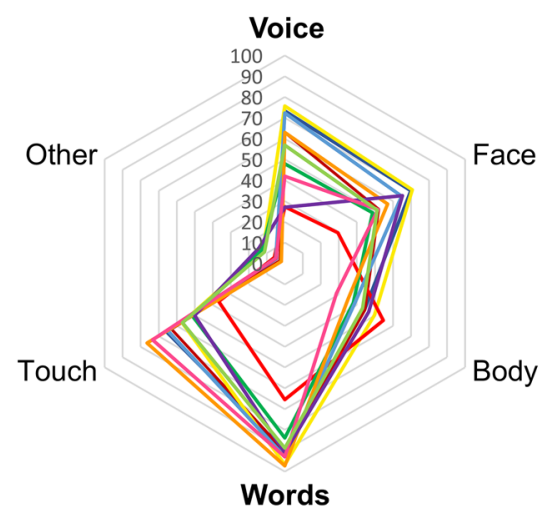

(D) Triumph

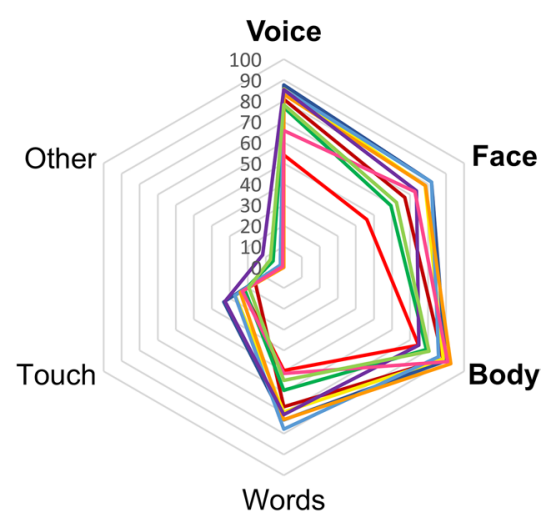

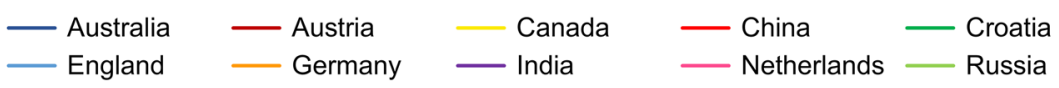

Fig. 2 Radial plots showing the percentage of participants reporting the use of different modalities to express a Feeling Moved, b Gratitude, c Interest, and d Triumph in ten countries. Note: The hypothesized modalities are indicated in bold

thought to be more frequently expressed via the face (reported by $70.92 \%$ participants overall), words (79.52\%), and voice $(55.15 \%)$, compared to the other modalities (16.59-36.64\%). Regarding these three most frequently used modalities, while the pairwise differences between them were not statistically significant in all countries, a clear pattern across the samples indicates that participants reported words the most frequently, the face slightly less frequently, and the voice the least frequently. 
Table 7 Support for Hypotheses per Country

\begin{tabular}{|c|c|c|c|c|}
\hline & $\begin{array}{c}\text { H1 } \\
\text { Feeling Moved } \\
\text { Face }>\text { Voice, } \\
\text { Body, Words, } \\
\text { Touch }\end{array}$ & $\begin{array}{c}\mathrm{H} 2 \\
\text { Gratitude } \\
\text { Voice, Words > } \\
\text { Face, Body, } \\
\text { Touch }\end{array}$ & $\begin{array}{c}\mathrm{H} 3 \\
\text { Interest } \\
\text { Voice, Face, } \\
\text { Words }>\text { Body, } \\
\text { Touch }\end{array}$ & $\begin{array}{c}\text { H4 } \\
\text { Triumph } \\
\text { Voice, Face, } \\
\text { Body > Words, } \\
\text { Touch }\end{array}$ \\
\hline Australia & $\checkmark$ & " & $\checkmark$ & 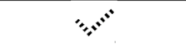 \\
\hline Austria & $\checkmark$ & 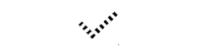 & $\checkmark$ & $\Rightarrow "$ \\
\hline Canada & $\checkmark$ & $\ddot{~}$ & $V$ & $\because "$ \\
\hline China & $x$ & $x$ & " & $\because$ \\
\hline Croatia & $\checkmark$ & " & $\checkmark$ & 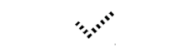 \\
\hline England & $\checkmark$ & $\because$ & $\checkmark$ & $x$ \\
\hline Germany & $\checkmark$ & " & $\checkmark$ & $\because "$ \\
\hline India & $\checkmark$ & " & $\because$ & " \\
\hline Netherlands & $V$ & $\because$ & ఊ" & $\because "$ \\
\hline Russia & $\checkmark$ & $\because$ & $\checkmark$ & 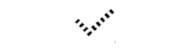 \\
\hline
\end{tabular}

Bold ticks indicate that the hypothesis was supported (all predicted modalities were significantly more frequently used than all other modalities). Dashed ticks indicate that the hypothesis was supported for at least one of the hypothesized modalities (i.e., at least one was significantly more frequently used than all nonhypothesized modalities). The crosses indicate that none of the hypothesized modalities were significantly more frequently used than non-hypothesized modalities

Overall, our hypothesis received strong support in terms of participants across countries reporting interest being expressed by words, while findings for the face and voice as expressions of interest were slightly less cross-culturally robust. Specifically, somewhat different trends emerged in two countries: In India, the voice did not stand out in frequency compared to the other modalities, while in China, this pertained to both the voice and face. Interestingly, touch emerged as a relatively frequently reported modality for expressing interest in China.

Triumph The results showed significant differences between selected modalities for expressions of triumph in all countries (see Table 8). In line with our hypothesis, participants across cultures thought triumph was more frequently expressed via the face (reported by $69.93 \%$ participants overall), body movement $(84.28 \%)$, and voice $(78.92 \%)$, as compared to touch $(24.70 \%)$. As in Studies 1a and 1b, words were consistently linked to expressing triumph across countries $(64.79 \%)$, not differing significantly in frequency compared to at least one of the hypothesized modalities in each country. In fact, in England, all three hypothesized modalities were not significantly different in reported frequency from 
Table 8 Cochran's Q statistics across ten countries for (a) feeling moved, (b) gratitude, (c) interest, and (d) triumph in Study 2

\begin{tabular}{|c|c|c|c|c|c|c|c|c|}
\hline \multirow[t]{2}{*}{ Country } & \multicolumn{4}{|c|}{ (A) Feeling moved } & \multicolumn{4}{|c|}{ (B) Gratitude } \\
\hline & $Q$ & $d f$ & $p$ & $R$ & $Q$ & $d f$ & $p$ & $R$ \\
\hline Australia & 368.74 & 5 & $<0.001$ & 0.290 & 400.95 & 5 & $<0.001$ & 0.306 \\
\hline Austria & 211.94 & 5 & $<0.001$ & 0.233 & 248.35 & 5 & $<0.001$ & 0.277 \\
\hline Canada & 335.17 & 5 & $<0.001$ & 0.304 & 327.13 & 5 & $<0.001$ & 0.283 \\
\hline China & 120.31 & 5 & $<0.001$ & 0.131 & 144.60 & 5 & $<0.001$ & 0.157 \\
\hline Croatia & 530.73 & 5 & $<0.001$ & 0.238 & 416.03 & 5 & $<0.001$ & 0.175 \\
\hline England & 359.07 & 5 & $<0.001$ & 0.296 & 387.19 & 5 & $<0.001$ & 0.315 \\
\hline Germany & 268.66 & 5 & $<0.001$ & 0.306 & 327.65 & 5 & $<0.001$ & 0.369 \\
\hline India & 187.97 & 5 & $<0.001$ & 0.213 & 224.69 & 5 & $<0.001$ & 0.242 \\
\hline Netherlands & 281.46 & 5 & $<0.001$ & 0.319 & 290.25 & 5 & $<0.001$ & 0.326 \\
\hline Russia & 192.03 & 5 & $<0.001$ & 0.184 & 226.11 & 5 & $<0.001$ & 0.206 \\
\hline \multirow[t]{2}{*}{ Country } & \multicolumn{4}{|c|}{ (C) Interest } & \multicolumn{4}{|c|}{ (D) Triumph } \\
\hline & $Q$ & $d f$ & $p$ & $R$ & $Q$ & $d f$ & $p$ & $R$ \\
\hline Australia & 438.25 & 5 & $<0.001$ & 0.352 & 558.51 & 5 & $<0.001$ & 0.447 \\
\hline Austria & 347.90 & 5 & $<0.001$ & 0.414 & 382.32 & 5 & $<0.001$ & 0.435 \\
\hline Canada & 402.63 & 5 & $<0.001$ & 0.380 & 472.55 & 5 & $<0.001$ & 0.435 \\
\hline China & 141.53 & 5 & $<0.001$ & 0.151 & 214.90 & 5 & $<0.001$ & 0.224 \\
\hline Croatia & 590.57 & 5 & $<0.001$ & 0.275 & 700.70 & 5 & $<0.001$ & 0.298 \\
\hline England & 487.71 & 5 & $<0.001$ & 0.418 & 537.26 & 5 & $<0.001$ & 0.454 \\
\hline Germany & 325.86 & 5 & $<0.001$ & 0.382 & 409.23 & 5 & $<0.001$ & 0.477 \\
\hline India & 283.66 & 5 & $<0.001$ & 0.332 & 269.07 & 5 & $<0.001$ & 0.286 \\
\hline Netherlands & 260.74 & 5 & $<0.001$ & 0.312 & 314.97 & 5 & $<0.001$ & 0.369 \\
\hline Russia & 246.67 & 5 & $<0.001$ & 0.248 & 318.11 & 5 & $<0.001$ & 0.306 \\
\hline
\end{tabular}

words as a means of expressing triumph. Again, while the pairwise differences between the most frequently used modalities were not statistically significant in all countries, a consistent pattern showed that participants on average reported body movement most frequently, followed by the voice, face, and words as signs of triumph. Body movement especially stood out in frequency in China and the Netherlands, where it was significantly more frequently reported for expressing triumph than all other modalities.

\section{Discussion}

In Study 2, we further mapped out how people report four specific positive emotions to be expressed, with samples from ten countries differing in terms of geographic region and cultural values. The results were highly consistent with those of Studies 1a and 1b, and across the cultural samples. As predicted, feeling moved was thought to be expressed via the face. This finding was consistent across all of the countries, except for China. For gratitude, words were, as hypothesised, deemed the most frequent expressions. Again, this finding was consistent across countries, with the exception of China. We did not find consistent evidence supporting the use of voice as an expressive modality for gratitude. For interest, 
words, face, and voice were deemed frequent expressions across countries, with some cultural specificities arising in India (where the voice was less frequent) and China (where the face and voice were less frequent than expected). Finally, across countries, triumph was thought to be expressed using facial, bodily, and vocal cues. Moreover, we replicated the finding from Studies $1 \mathrm{a}$ and $1 \mathrm{~b}$ showing that participants thought that triumph was frequently expressed with words.

\section{General Discussion}

In three studies, we examined how feeling moved, gratitude, interest, and triumph are thought to be expressed, by adopting a variant of self-report: the intersubjective approach (Chiu et al., 2010). We show that people think that feeling moved is primarily expressed on the face, gratitude with the use of words, interest via words, face, and voice, and triumph with body postures, vocal cues, facial expressions, and words. In Study 1a we found evidence in a U.S.A. sample in favour of our exploratory hypotheses driven by previous empirical work (Keltner \& Cordaro, 2017) and theoretical considerations (Sauter, 2017). In Study $1 \mathrm{~b}$ we replicated this general pattern of results, even when participants were not provided with emotion definitions. In Study 2 we engaged in a cross-cultural comparison spanning ten countries that diverged in cultural values (Minkov \& Hofstede, 2012; Schwartz et al., 2001). Associations between expressive patterns and emotions were mostly replicated, although several culture-specific findings also surfaced. We also note that across samples, every modality was reported to be used for expressing each emotion; this aligns with evidence suggesting that positive emotions are expressed through multiple communicative channels (Keltner \& Cordaro, 2017). These findings contribute to the growing literature on positive emotion expressions (Shiota et al., 2017), and also demonstrates the utility of a methodological tool that could aid in conducting further cross-cultural work with community samples.

\section{Cross-Culturally Consistent Results}

The present findings provide empirical evidence that highlights contrasting use of different modalities for expressing feeling moved, gratitude, interest, and triumph. These findings were largely replicated across the cultural contexts, and align with theorising about the functions of positive emotions both from the emotion family perspective (Sauter, 2017) and the arousal-engagement matrix (Kitayama et al., 2006; Tsai et al., 2006). The cross-cultural consistency of our findings suggests statistical regularities in the social environments of participants that arguably reflect actually occurring expressions, thereby pointing to potential consistencies in how people from varied cultures express specific positive emotions. To establish whether these patterns are present in actual behaviour will require further study.

Across cultures, participants consistently reported that feeling moved is expressed via the face. This is in line with work suggesting that tears (Schubert et al., 2018; Seibt et al., 2018) are consistent markers of this emotion. Tears signal being overwhelmed (Gracanin et al., 2018), which is a key component of feeling moved. The present findings also align with the high-arousal high-engagement nature of displaying feeling moved. Tears are associated with states of high arousal when experiencing feeling moved, and signal a need for help-thereby drawing others closer for social engagement. 
Participants thought that gratitude is primarily expressed with words, as we had hypothesized. However, although we also expected the voice to be used frequently to express gratitude, this was not supported by our findings. The voice was not reported as often as were words, nor was the voice any different in frequency from the face and touch, thereby suggesting that the voice is not a salient a modality for expressing gratitude (see also Floyd et al., 2018). Touch emerged as a quite frequent modality for expressing gratitude, and in some cases (such as the Netherlands and Germany), was second only to words. These findings are in line with past theorising on touch being a key modality for affiliative gestures (App et al., 2011). We did not hypothesize touch as a critical modality given the contextunspecified nature of our measure. We expected touch to emerge as a modality used to express gratitude only when the perceiver was deemed a close other, since touch could also be viewed as a violation of ones' privacy and space (Lee \& Guerrero, 2001). Even though participants made judgments about members of their group in general (without a context specified), touch was often thought to be used for expressing gratitude. This aligns with the expectation that expressions of prosocial positive emotions would include the recruitment of touch.

For interest, words emerged as the most often used modality, with the face also commonly reported. The voice was recruited in many cultures as well, but this finding was less cross-culturally robust than the highly consistent findings for the use of words to express interest. In line with theorising about epistemological emotions, our findings highlight the crucial nature words play in conveying a shift or update in ones' knowledge state. Furthermore, the low-arousal nature of interest seems well reflected in the fact that bodily movement was not frequently reported as a modality used to express interest, even though some empirical work has suggested that such expressions can occur (Dael et al., 2012a, 2012b).

For expressing triumph, body posture, voice, and the face were all deemed as frequently used modalities, in line with past empirical and theoretical work suggesting that dominance expressions are signalled with expansive gestures that recruit multiple modalities (App et al., 2011; Van Osch et al., 2013). What was surprising, however, was that participants across cultures reported words to be one of the primary ways to express triumph. We initially thought these findings may be specific to the U.S.A., where explicit verbal expressions of victory are encouraged (Hwang et al., 2016). However, this result was consistent across cultural groups, and potentially reflects the fact that our measure did not specify a particular time point for expression. Perhaps participants were thinking about what happens in the aftermath of a victory as well, when social sharing of emotions is common (Rime, 2009).

\section{Culture-Specific Patterns}

Our results also point to some culture specific patterns, although it is worth noting that the statistical tests employed were not set up to directly test cross-cultural variability. For example, baseline differences in how many modalities people from divergent cultures select can yield cultural differences. Indeed, the Chinese sample had overall lower response frequencies as compared to the other samples. Nonetheless, we descriptively map out the key cultural specificities found in our data and point to potential theoretical reasons for why these patterns may have been found. ${ }^{6}$

\footnotetext{
${ }^{6}$ Following the approach of comparing the two U.S.A. samples, we also investigated differences between the ten countries in absolute levels of endorsement of each modality, for each emotion. These results can be found in the Supplementary Materials (ESM Pg. 17-31).
} 
For feeling moved, we did not obtain support in favour of our hypothesis in China, where words were reported as often as the face to express feeling moved. One possible explanation is the existence of strict facial display rules in China. Strong cultural norms might constrict the outward expression of emotions that signal vulnerability via tears (Davis et al., 2012). Previous research has shown strong display rules for expressing sadness in East Asia (Matsumoto et al., 2008), which could generalise to other forms of tearbased expressions as well. In this cultural context, individuals might rely more on words to communicate feeling moved to others.

An interesting pattern emerged for gratitude expressions in India, where the voice was thought to be least frequently used. Unexpectedly, the face, body and touch were all reported to be more frequently used than the voice. These findings could have to do with the relational norms that are prevalent in Indian society, where kinship ties are maintained and fostered through reciprocity and the open expressions of thankfulness (Miller et al., 2017). As such, words could be used alongside other modalities that quite clearly signal being thankful in this cultural context-including the face-which is a primary conveyor of social information and is often used to create and sustain affiliation (Van Kleef, 2009).

Findings for interest in China raise key theoretical questions regarding the classification of interest as a primarily inward-looking (or socially disengaging) emotion (see also Sung $\&$ Yih, 2016). In the Chinese context, touch (which could be conceptualised as an affiliative gesture; App et al., 2011) was also recruited for the expression of interest. Perhaps in some cultural contexts, interest could be conceptualised as a socially engaging emotion that is primarily relevant with reference to other people (Silvia, 2005). This line of argumentation fits recent arguments pointing to the role of context in determining the degree to which interest is inward-focused (see also Greenaway et al., 2018).

\section{Strengths, Limitations, and Future Directions}

A key strength of the present research is that we examined expressions from the perspective of the perceiver, thereby circumventing the need for and bias of introspective judgments. Accurate introspection by participants should not be assumed, particularly when expressions can be quick and fleeting, possibly even beyond the threshold of consciousness (Yan et al., 2013). Our use of the intersubjective approach is based not on reports about oneself, but on observations of others, which helps mitigate some of these concerns. Furthermore, the fact that our findings align with the available empirical literature on emotional expressions predominantly conducted in the U.S.A. (Cowen \& Keltner, 2020), suggests that our choice of method is appropriate.

Potential limitations of our work relate specifically to the cross-cultural nature of our research. If measures from individuals are considered a reflection of a country's response patterns, then a large representative sample would be required for an accurate representation of that country. Such sample features may not be achieved if researchers recruit paid participants from online platforms that are microcosms in themselves (see Chmielewski \& Kucker, 2020), or hard-to-obtain populations using a convenience snowballing approach.

Furthermore, when using rating scales to contrast between groups, culturally dependent response bias is likely to occur. A growing literature has demonstrated that cultural norms influence response styles in Likert-type items, such as acquiescence bias in countries where deference to authority is valued (e.g., East Asia: Chen et al., 1995), and extreme responding in cultures where amplified expressions are encouraged (e.g., Latin America: Culpepper \& Zimmerman, 2006). Such biases undermine the reliability 
of conclusions researchers can draw from cross-cultural comparisons of intersubjective measures, as genuine differences become conflated with response styles.

The methods we employed deal with these considerations in two main ways. Rather than assuming participants to be prototypical or representative of their culture, the intersubjective approach taps into the shared knowledge and expertise of group members; respondents are hence viewed as experts of their culture due to their lived experiences (Wan et al., 2010). As such, a culture's pattern of responses is inferred from the degree of agreement between raters. Second, rather than using classic rating scales, we provide categorical response options that are clearly labelled in participants' native language. We also provided emotion definitions that were closely matched across the languages because we judged it essential to maintain clarity. This could potentially have biased respondents to select particular modalities. The replication of our US results in Study $1 \mathrm{~b}$ (without definitions) indicates that participants' judgments were not driven by the definitions, but we cannot rule this possibility out empirically for the other samples.

While intersubjectivity has many benefits, we also acknowledge possible downsides of this method. Even with the adjustments mentioned above, some caution needs be exercised when interpreting cultural differences, particularly due to variance between samples that may be explained by factors other than national culture. As we used a variety of approaches to collect data, our samples were not fully matched, and could potentially differ on characteristics such as socioeconomic status or religious orientation (see Cohen, 2009). Another key contributor to cultural differences is language (Kashima \& Kashima, 1998). While we used an established method for translating all materials (team-translation approach: Clark et al., 2017), the possibility of language differences contributing to cultural differences on our measures cannot be ruled out.

Furthermore, this type of measure taps into peoples' shared understanding of others' expressions, which means that our results could reflect consensually shared stereotypes rather than actually occurring expressions. Stereotypes about emotional reactions are likely to reflect, at least in part, participants' everyday observations. The fact that our results were largely consistent across 11 very different samples thus points to possible similarities in actually occurring behaviors. Another limitation of our measure is the level of granularity that our items tap into. For example, while 'touch' was provided as a modality option, our research tool was unable to differentiate between different forms of touch. In reality, a pat on the shoulder is of course hugely different from a slap in the face. Future work may refine the present tool to provide greater detail for each modality, perhaps with branch logic such that finer granularity is achieved within each option.

In a related vein, we recommend several lines of future research that could unpack why and how cultural differences manifest in the expression of positive emotions. We here point to two culture specific patterns we observed in our Chinese sample. First, Chinese participants overall selected fewer modalities in comparison to other countries, and second, we found culture specific patterns for expressions of feeling moved and gratitude. A possible explanation for the above findings could be cultural display rules, where some positive emotions are deemed less acceptable to express as compared to others (see Manokara et al., 2021). Future research could focus on cross-cultural comparisons, by blending classic production methods with the intersubjective approach, to understand whether the patterns we find reflect genuine cultural differences in emotion expressions, or mere differences in cultural representations.

Another extension for future work would be to examine the role of context in shaping emotion expressions. Incorporating features of the situation has been put forth as a recommendation for classic production studies as well (Greenaway et al., 2018), and researchers 
could start by empirically testing whether contextual information shapes self-reported emotion expressions, before investing greater resources in lab studies. We provide a tool that is flexible enough to allow for modification (see OSF for translated materials), as a first step in examining novel research questions.

\section{Conclusion}

In our present research, we demonstrate that people across a range of divergent cultures think that some positive emotions (feeling moved, gratitude, interest, triumph) map onto modality-specific expressive patterns. In line with past theorising and empirical work, feeling moved was associated with expressions via the face, gratitude was expressed with words, interest displays involved words, the face as well as the voice, and triumph was expressed via body postures, vocal and facial expressions, and words. Our findings thus provide an initial empirical grounding for further theorising on positive emotions. We mapped out the above patterns using the intersubjective approach (Chiu et al., 2010), thereby introducing a methodological tool that we hope will facilitate further cross-cultural work on nonverbal behaviour.

Supplementary Information The online version contains supplementary material available at https://doi. org/10.1007/s10919-021-00376-0.

Acknowledgements We would like to thank Evgeniya Vedernikova, Alice Mayer-Heinsich, Chuting Cao, and Ketaki A. Diwan, for collecting data in Russia, Austria, China, and India, respectively. We confirm that the manuscript is not under review elsewhere, that the primary data have not been published previously or accepted for publication, and that the appropriate ethical guidelines were followed in the conduct of our research. This research was funded by a Starting Grant from the European Research Council, awarded to the senior author.

Open Access This article is licensed under a Creative Commons Attribution 4.0 International License, which permits use, sharing, adaptation, distribution and reproduction in any medium or format, as long as you give appropriate credit to the original author(s) and the source, provide a link to the Creative Commons licence, and indicate if changes were made. The images or other third party material in this article are included in the article's Creative Commons licence, unless indicated otherwise in a credit line to the material. If material is not included in the article's Creative Commons licence and your intended use is not permitted by statutory regulation or exceeds the permitted use, you will need to obtain permission directly from the copyright holder. To view a copy of this licence, visit http://creativecommons.org/licenses/by/4.0/.

\section{References}

Algoe, S. B. (2012). Find, remind, and bind: The functions of gratitude in everyday relationships. Social and Personality Psychology Compass, 6(6), 455-469.

Algoe, S. B., Fredrickson, B. L., \& Gable, S. L. (2013). The social functions of the emotion of gratitude via expression. Emotion, 13(4), 605-609.

Algoe, S. B., \& Haidt, J. (2009). Witnessing excellence in action: The 'other-praising'emotions of elevation, gratitude, and admiration. The Journal of Positive Psychology, 4(2), 105-127.

Ambadar, Z., Cohn, J. F., \& Reed, L. I. (2009). All smiles are not created equal: Morphology and timing of smiles perceived as amused, polite, and embarrassed/nervous. Journal of Nonverbal Behavior, 33(1), $17-34$.

App, B., McIntosh, D. N., Reed, C. L., \& Hertenstein, M. J. (2011). Non- verbal channel use in communication of emotion: How may depend on why. Emotion, 11(3), 603-617. 
Banse, R., \& Scherer, K. R. (1996). Acoustic profiles in vocal emotion expression. Journal of Personality and Social Psychology, 70(3), 614-636.

Berry, K. J., Johnston, J. E., \& Mielke, P. W., Jr. (2007). An alternative measure of effect size for Cochran's $\mathrm{Q}$ test for related proportions. Perceptual and Motor Skills, 104(3), 1236-1242.

Boiger, M., \& Mesquita, B. (2012). The construction of emotion in interactions, relationships, and cultures. Emotion Review, 4(3), 221-229.

Campos, B., Shiota, M. N., Keltner, D., Gonzaga, G. C., \& Goetz, J. L. (2013). What is shared, what is different? Core relational themes and expressive displays of eight positive emotions. Cognition \& Emotion, 27(1), 37-52.

Chen, C., Lee, S. Y., \& Stevenson, H. W. (1995). Response style and cross-cultural comparisons of rating scales among East Asian and North American students. Psychological Science, 6(3), 170-175.

Chiu, C. Y., Gelfand, M. J., Yamagishi, T., Shteynberg, G., \& Wan, C. (2010). Intersubjective culture: The role of intersubjective perceptions in cross-cultural research. Perspectives on Psychological Science, 5(4), 482-493.

Chmielewski, M., \& Kucker, S. C. (2020). An MTurk crisis? Shifts in data quality and the impact on study results. Social Psychological and Personality Science, 11(4), 464-473.

Clark, L., Birkhead, A. S., Fernandez, C., \& Egger, M. J. (2017). A transcription and translation protocol for sensitive cross-cultural team research. Qualitative Health Research, 27(12), 1751-1764.

Cochran, W. G. (1950). The comparison of percentages in matched samples. Biometrika, 37(3/4), $256-266$.

Cohen, A. B. (2009). Many forms of culture. American Psychologist, 64(3), 194-204.

Cordaro, D. T., Sun, R., Keltner, D., Kamble, S., Huddar, N., \& McNeil, G. (2018). Universals and cultural variations in 22 emotional expressions across five cultures. Emotion, 18(1), 75-93.

Cova, F., \& Deonna, J. A. (2014). Being moved. Philosophical Studies, 169(3), 447-466.

Cowen, A. S., \& Keltner, D. (2020). What the face displays: Mapping 28 emotions conveyed by naturalistic expression. American Psychologist, 75(3), 349-364.

Cowen, A. S., Elfenbein, H. A., Laukka, P., \& Keltner, D. (2019). Mapping 24 emotions conveyed by brief human vocalization. American Psychologist, 74(6), 698-712.

Culpepper, R. A., \& Zimmerman, R. A. (2006). Culture-based extreme response bias in surveys employing variable response items: An investigation of response tendency among Hispanic-Americans. Journal of International Business Research, 5(2), 75-83.

Dael, N., Mortillaro, M., \& Scherer, K. R. (2012a). Emotion expression in body action and posture. Emotion, 12(5), 1085.

Dael, N., Mortillaro, M., \& Scherer, K. R. (2012b). The body action and posture coding system (BAP): Development and reliability. Journal of Nonverbal Behavior, 36(2), 97-121.

Davis, E., Greenberger, E., Charles, S., Chen, C., Zhao, L., \& Dong, Q. (2012). Emotion experience and regulation in China and the United States: How do culture and gender shape emotion responding? International Journal of Psychology, 47(3), 230-239.

Dukes, D., Clément, F., Audrin, C., \& Mortillaro, M. (2017). Looking beyond the static face in emotion recognition: The informative case of interest. Visual Cognition, 25(4-6), 575-588.

Ekman, P. (1992). An argument for basic emotions. Cognition \& Emotion, 6(3-4), 169-200.

Ekman, P. (1994). Strong evidence for universals in facial expressions: A reply to Russell's mistaken critique. Psychological Bulletin, 115(2), 268-287.

Ekman, P., Friesen, W. V., \& Hager, J. V. (2002). Facial action coding system (2nd ed.). Research Nexus eBook.

Ekman, P., Friesen, W. V., O'sullivan, M., Chan, A., Diacoyanni-Tarlatzis, I., Heider, K., \& Scherer, K. (1987). Universals and cultural differences in the judgments of facial expressions of emotion. Journal of Personality and Social Psychology, 53(4), 712-717.

Elfenbein, H. A., Beaupré, M., Lévesque, M., \& Hess, U. (2007). Toward a dialect theory: Cultural differences in the expression and recognition of posed facial expressions. Emotion, 7(1), 131-146.

Everitt, B. S. (1975). Multivariate analysis: The need for data, and other problems. The British Journal of Psychiatry, 126(3), 237-240.

Faul, F., Erdfelder, E., Buchner, A., \& Lang, A.-G. (2009). Statistical power analyses using G*Power 3.1: Tests for correlation and regression analyses. Behavior Research Methods, 41(4), 1149-1160.

Fiske, A. P., Seibt, B., \& Schubert, T. (2019). The sudden devotion emotion: Kama muta and the cultural practices whose function is to evoke it. Emotion Review, 11(1), 74-86.

Floyd, S., Rossi, G., Baranova, J., Blythe, J., Dingemanse, M., Kendrick, K. H., \& Enfield, N. J. (2018). Universals and cultural diversity in the expression of gratitude. Royal Society Open Science, 5(5), 180391.

Fredrickson, B. L. (2001). The role of positive emotions in positive psychology: The broaden-and-build theory of positive emotions. American Psychologist, 56(3), 218-226. 
Fridlund, A. J. (1994). Human facial expression: An evolutionary view. Academic Press.

Goffman, E. (1959). The presentation of self in everyday life. Doubleday.

Gračanin, A., Bylsma, L. M., \& Vingerhoets, A. J. (2018). Why only humans shed emotional tears. Human Nature, 29(2), 104-133.

Greenaway, K. H., Kalokerinos, E. K., \& Williams, L. A. (2018). Context is everything (in emotion research). Social and Personality Psychology Compass, 12(6), e12393.

Henrich, J., Heine, S. J., \& Norenzayan, A. (2010). Most people are not WEIRD. Nature, 466(7302), 29-29.

Hertenstein, M. J., Holmes, R., McCullough, M., \& Keltner, D. (2009). The communication of emotion via touch. Emotion, 9(4), 566-573.

Hervé, M. (2020). RVAideMemoire: Testing and Plotting Procedures for Biostatistics. R package version 0.9-77. https://CRAN.R-project.org/package=RVAideMemoire

Hwang, H. C., \& Matsumoto, D. (2014). Cultural differences in victory signals of triumph. Cross-Cultural Research, 48(2), 177-191.

Hwang, H. C., Matsumoto, D., Yamada, H., Kostić, A., \& Granskaya, J. V. (2016). Self-reported expression and experience of triumph across four countries. Motivation and Emotion, 40(5), 731-739.

Kalokerinos, E. K., Greenaway, K. H., Pedder, D. J., \& Margetts, E. A. (2014). Don't grin when you win: The social costs of positive emotion expression in performance situations. Emotion, 14(1), 180-186.

Kashima, E. S., \& Kashima, Y. (1998). Culture and language: The case of cultural dimensions and personal pronoun use. Journal of Cross-Cultural Psychology, 29(3), 461-486.

Keltner, D. (2019). Toward a consensual taxonomy of emotions. Cognition \& Emotion, 33(1), 14-19.

Keltner, D., \& Cordaro, D. T. (2017). Understanding multimodal emotional expressions: Recent advances in basic emotion theory. In J. M. Fernandez-Dols \& J. A. Russell (Eds.), The science of facial expression (pp. 57-76). Oxford University Press.

Kessous, L., Castellano, G., \& Caridakis, G. (2010). Multimodal emotion recognition in speech-based interaction using facial expression, body gesture and acoustic analysis. Journal on Multimodal User Interfaces, 3(1-2), 33-48.

Kitayama, S., Markus, H. R., \& Kurokawa, M. (2000). Culture, emotion, and well-being: Good feelings in Japan and the United States. Cognition \& Emotion, 14(1), 93-124.

Kitayama, S., Mesquita, B., \& Karasawa, M. (2006). Cultural affordances and emotional experience: Socially engaging and disengaging emotions in Japan and the United States. Journal of Personality and Social Psychology, 91(5), 890.

Krumhuber, E., Küster, D., Namba, S., Shah, D., \& Calvo, M. G. (2019). Emotion recognition from posed and spontaneous dynamic expressions: Human observers vs. machine analysis. Emotion. https://doi. org/10.1037/emo0000712

Larsen, R. J., \& Diener, E. (1992). Promises and problems with the circumplex model of emotion. In M. S. Clark (Ed.), Review of personality and social psychology: Emotion (pp. 25-59). Sage.

Laukka, P. (2005). Categorical perception of vocal emotion expressions. Emotion, 5(3), 277.

LeDoux, J. E., \& Brown, R. (2017). A higher-order theory of emotional consciousness. Proceedings of the National Academy of Sciences, 114(10), E2016-E2025.

Lee, J. W., \& Guerrero, L. K. (2001). Types of touch in cross-sex relationships between coworkers: Perceptions of relational and emotional messages, inappropriateness, and sexual harassment. Journal of Applied Communication Research, 29(3), 197-220.

Levenson, R. W., Carstensen, L. L., Friesen, W. V., \& Ekman, P. (1991). Emotion, physiology, and expression in old age. Psychology and Aging, 6(1), 28-35.

Mangiafico, S. (2020). rcompanion: Functions to Support Extension Education Program Evaluation. R package version 2.3.25. https://CRAN.R-project.org/package=rcompanion

Manokara, K., Fischer, A., \& Sauter, D. (2021). Display rules differ between positive emotions: Not all that feels good, looks good. https://doi.org/10.31219/osf.io/4uaym

Manokara, K., \& Sauter, D. A. (2021). Emotion universals: The foundations from which cultural variability of emotion emerges. In J. De Leersnyder (Ed.), The sociocultural shaping of emotion. Cambridge University Press.

Matsumoto, D. (1990). Cultural similarities and differences in display rules. Motivation and Emotion, 14(3), 195-214.

Matsumoto, D., \& Hwang, H. S. (2012). Evidence for a nonverbal expression of triumph. Evolution and Human Behavior, 33(5), 520-529.

Matsumoto, D., Yoo, S. H., \& Fontaine, J. (2008). Mapping expressive differences around the world: The relationship between emotional display rules and individualism versus collectivism. Journal of CrossCultural Psychology, 39(1), 55-74.

Menninghaus, W., Wagner, V., Hanich, J., Wassiliwizky, E., Kuehnast, M., \& Jacobsen, T. (2015). Towards a psychological construct of being moved. PLOS ONE, 10(6), e0128451. 
Mielke, P. W., Jr., Berry, K. J., \& Johnston, J. E. (2007). Resampling programs for multiway contingency tables with fixed marginal frequency totals. Psychological Reports, 101(1), 18-24.

Miller, J. G., Akiyama, H., \& Kapadia, S. (2017). Cultural variation in communal versus exchange norms: Implications for social support. Journal of Personality and Social Psychology, 113(1), 81.

Minkov, M., \& Hofstede, G. (2012). Hofstede's fifth dimension: New evidence from the World Values Survey. Journal of Cross-Cultural Psychology, 43(1), 3-14.

Mortillaro, M., Mehu, M., \& Scherer, K. R. (2011). Subtly different positive emotions can be distinguished by their facial expressions. Social Psychological and Personality Science, 2(3), 262-271.

Nummenmaa, L., Glerean, E., Hari, R., \& Hietanen, J. K. (2014). Bodily maps of emotions. Proceedings of the National Academy of Sciences, 111(2), 646-651.

R Core Team. (2019). R: A language and environment for statistical computing. R Foundation for Statistical Computing, Vienna, Austria. URL https://www.R-project.org/

Reeve, J. (1993). The face of interest. Motivation and Emotion, 17(4), 353-375.

Rimé, B. (2009). Emotion elicits the social sharing of emotion: Theory and empirical review. Emotion Review, $1(1), 60-85$.

Roseman, I. J. (1996). Appraisal determinants of emotions: Constructing a more accurate and comprehensive theory. Cognition \& Emotion, 10(3), 241-278.

Rozin, P., Taylor, C., Ross, L., Bennett, G., \& Hejmadi, A. (2005). General and specific abilities to recognise negative emotions, especially disgust, as portrayed in the face and the body. Cognition \& Emotion, 19(3), $397-412$.

Russell, J. A. (1994). Is there universal recognition of emotion from facial expression? A review of the crosscultural studies. Psychological Bulletin, 115(1), 102-141.

Sauter, D. A. (2017). The nonverbal communication of positive emotions: An emotion family approach. Emotion Review, 9(3), 222-234.

Sauter, D. A., Eisner, F., Ekman, P., \& Scott, S. K. (2010). Cross-cultural recognition of basic emotions through nonverbal emotional vocalizations. Proceedings of the National Academy of Sciences of the United States of America, 107(6), 2408-2412.

Sauter, D. A., \& Russell, J. A. (2020). What do nonverbal expressions tell us about emotion? In A. Scarantino (Ed.), Handbook of emotion theory. Taylor and Francis.

Sauter, D. A., \& Scott, S. K. (2007). More than one kind of happiness: Can we recognize vocal expressions of different positive states? Motivation and Emotion, 31(3), 192-199.

Schirmer, A., \& Adolphs, R. (2017). Emotion perception from face, voice, and touch: Comparisons and convergence. Trends in Cognitive Sciences, 21(3), 216-228.

Schubert, T. W., Zickfeld, J. H., Seibt, B., \& Fiske, A. P. (2018). Moment-to-moment changes in feeling moved match changes in closeness, tears, goosebumps, and warmth: Time series analyses. Cognition \& Emotion, 32(1), 174-184.

Schuller, D. M., \& Schuller, B. W. (2020). A review on five recent and near-future developments in computational processing of emotion in the human voice. Emotion Review. https://doi.org/10.1177/1754073919 898526

Schwartz, S. H., Melech, G., Lehmann, A., Burgess, S., Harris, M., \& Owens, V. (2001). Extending the crosscultural validity of the theory of basic human values with a different method of measurement. Journal of Cross-Cultural Psychology, 32(5), 519-542.

Seibt, B., Schubert, T. W., Zickfeld, J. H., Zhu, L., Arriaga, P., Simão, C., \& Fiske, A. P. (2018). Kama muta: Similar emotional responses to touching videos across the United States, Norway, China, Israel, and Portugal. Journal of Cross-Cultural Psychology, 49(3), 418-435.

Serlin, R. C., Carr, J., \& Marascuilo, L. A. (1982). A measure of association for selected nonparametric procedures. Psychological Bulletin, 92(3), 786.

Shaver, P., Schwartz, J., Kirson, D., \& O’Connor, C. (1987). Emotion knowledge: Further explorations of a prototype approach. Journal of Personality and Social Psychology, 52(6), 1061-1086.

Shiota, M. N., Campos, B., \& Keltner, D. (2003). The faces of positive emotion: Prototype displays of awe, amusement, and pride. Annals of the New York Academy of Sciences, 1000(1), 296-299.

Shiota, M. N., Campos, B., Oveis, C., Hertenstein, M. J., Simon-Thomas, E., \& Keltner, D. (2017). Beyond happiness: Building a science of discrete positive emotions. American Psychologist, 72(7), 617-643.

Silvia, P. J. (2005). What is interesting? Exploring the appraisal structure of interest. Emotion, 5(1), 89-102.

Silvia, P. J. (2008). Interest-The curious emotion. Current Directions in Psychological Science, 17(1), 57-60.

Simon-Thomas, E. R., Keltner, D. J., Sauter, D., Sinicropi-Yao, L., \& Abramson, A. (2009). The voice conveys specific emotions: Evidence from vocal burst displays. Emotion, 9(6), 838-846.

Sperber, A. D., Devellis, R. F., \& Boehlecke, B. (1994). Cross-cultural translation: Methodology and validation. Journal of Cross-Cultural Psychology, 25(4), 501-524. 
Sung, B., \& Yih, J. (2016). Does interest broaden or narrow attentional scope? Cognition and Emotion, 30(8), 1485-1494.

Tiedens, L. Z. (2001). Anger and advancement versus sadness and subjugation: The effect of negative emotion expressions on social status conferral. Journal of Personality and Social Psychology, 80(1), 86-94.

Tooby, J., \& Cosmides, L. (2008). The evolutionary psychology of the emotions and their relationship to internal regulatory variables. In M. Lewis, J. M. Haviland-Jones, \& L. F. Barrett (Eds.), Handbook of emotions (pp. 114-137). Guilford Press.

Tracy, J. L., \& Matsumoto, D. (2008). The spontaneous expression of pride and shame: Evidence for biologically innate nonverbal displays. Proceedings of the National Academy of Sciences, 105(33), 11655-11660.

Tracy, J. L., \& Robins, R. W. (2007). The prototypical pride expression: Development of a nonverbal behavior coding system. Emotion, 7(4), 789-801.

Tracy, J. L., \& Robins, R. W. (2008). The nonverbal expression of pride: Evidence for cross-cultural recognition. Journal of Personality and Social Psychology, 94(3), 516-530.

Tsai, J. L., Knutson, B., \& Fung, H. H. (2006). Cultural variation in affect valuation. Journal of Personality and Social Psychology, 90(2), 288-307.

Van Cappellen, P., \& Edwards, M. E. (2020). The embodiment of worship: Relations among postural, psychological, and physiological aspects of religious practice. Journal for the Cognitive Science of Religion, 6 , 56.

Van Herk, H., Poortinga, Y. H., \& Verhallen, T. M. (2004). Response styles in rating scales: Evidence of method bias in data from six EU countries. Journal of Cross-Cultural Psychology, 35(3), 346-360.

Van Kleef, G. A. (2009). How emotions regulate social life: The emotions as social information (EASI) model. Current Directions in Psychological Science, 18(3), 184-188.

Van Osch, Y., Zeelenberg, M., Breugelmans, S. M., \& Brandt, M. J. (2019). Show or hide pride? Selective inhibition of pride expressions as a function of relevance of achievement domain. Emotion, 19(2), 334-347.

Van Osch, Y. M. J., Breugelmans, S. M., Zeelenberg, M., \& Fontaine, J. J. R. (2013). The meaning of pride across cultures. In J. J. R. Fontaine, K. R. Scherer, \& C. Soriano (Eds.), Series in affective science: Components of emotional meaning: A sourcebook (pp. 377-387). Oxford University Press.

Vrticka, P., Lordier, L., Bediou, B., \& Sander, D. (2014). Human amygdala response to dynamic facial expressions of positive and negative surprise. Emotion, 14(1), 161-169.

Vuoskoski, J. K., \& Eerola, T. (2017). The pleasure evoked by sad music is mediated by feelings of being moved. Frontiers in Psychology, 8, 439.

Wan, C., Torelli, C. J., \& Chiu, C. Y. (2010). Intersubjective consensus and the maintenance of normative shared reality. Social Cognition, 28(3), 422-446.

Wassiliwizky, E., Jacobsen, T., Heinrich, J., Schneiderbauer, M., \& Menninghaus, W. (2017a). Tears falling on goosebumps: Co- occurrence of emotional lacrimation and emotional piloerec- tion indicates a psychophysiological climax in emotional arousal. Frontiers in Psychology. https://doi.org/10.3389/fpsyg.2017. 00041

Wassiliwizky, E., Koelsch, S., Wagner, V., Jacobsen, T., \& Menninghaus, W. (2017b). The emotional power of poetry: Neural circuitry, psychophysiology and compositional principles. Social Cognitive and Affective Neuroscience, 12(8), 1229-1240.

Williams, L. A., \& Bartlett, M. Y. (2015). Warm thanks: Gratitude expression facilitates social affiliation in new relationships via perceived warmth. Emotion, 15(1), 1-5.

Yan, W. J., Wu, Q., Liang, J., Chen, Y. H., \& Fu, X. (2013). How fast are the leaked facial expressions: The duration of micro-expressions. Journal of Nonverbal Behavior, 37(4), 217-230.

Yoo, S. H., \& Noyes, S. E. (2016). Recognition of facial expressions of negative emotions in romantic relationships. Journal of Nonverbal Behavior, 40(1), 1-12.

Yoon, J., Desmet, P. M., \& van der Helm, A. (2012). Design for interest: Exploratory study on a distinct positive emotion in human-product interaction. International Journal of Design, 6(2), 67-80.

Yoshimura, S. M., \& Berzins, K. (2017). Grateful experiences and expressions: The role of gratitude expressions in the link between gratitude experiences and well-being. Review of Communication, 17(2), $106-118$.

Zickfeld, J. H., Arriaga, P., Santos, S. V., Schubert, T. W., \& Seibt, B. (2020). Tears of joy, aesthetic chills and heartwarming feelings: Physiological correlates of Kama Muta. Psychophysiology, 57(12), e13662.

Zickfeld, J. H., Schubert, T. W., Seibt, B., Blomster, J. K., Arriaga, P., Basabe, N., \& Ding, Y. (2019b). Kama muta: Conceptualizing and measuring the experience often labelled being moved across 19 nations and 15 languages. Emotion, 19(3), 402-425.

Zickfeld, J. H., Schubert, T. W., Seibt, B., \& Fiske, A. P. (2019a). Moving through the literature: What is the emotion often denoted being moved? Emotion Review, 11(2), 123-139. 
Publisher's Note Springer Nature remains neutral with regard to jurisdictional claims in published maps and institutional affiliations. 